\title{
Non-Self-Adjoint Toeplitz Matrices Whose Principal Submatrices Have Real Spectrum
}

\author{
Boris Shapiro $^{1}$ • František Štampach ${ }^{1}$
}

Received: 29 March 2017 / Revised: 28 August 2017 / Accepted: 5 October 2017 /

Published online: 7 December 2017

(C) The Author(s) 2017. This article is an open access publication

\begin{abstract}
We introduce and investigate a class of complex semi-infinite banded Toeplitz matrices satisfying the condition that the spectra of their principal submatrices accumulate onto a real interval when the size of the submatrix grows to $\infty$. We prove that a banded Toeplitz matrix belongs to this class if and only if its symbol has real values on a Jordan curve located in $\mathbb{C} \backslash\{0\}$. Surprisingly, it turns out that, if such a Jordan curve is present, the spectra of all the principal submatrices have to be real. The latter claim is also proved for matrices given by a more general symbol. The special role of the Jordan curve is further demonstrated by a new formula for the limiting density of the asymptotic eigenvalue distribution for banded Toeplitz matrices from the studied class. Certain connections between the problem under investigation, Jacobi operators, and the Hamburger moment problem are also discussed. The main results are illustrated by several concrete examples; some of them allow an explicit analytic treatment, while some are only treated numerically.
\end{abstract}

Keywords Banded Toeplitz matrix - Asymptotic eigenvalue distribution - Real spectrum · Non-self-adjoint matrices · Moment problem · Jacobi matrices · Orthogonal polynomials

Mathematics Subject Classification 15B05 - 47B36 · 33C47

Communicated by Serguei Denissov.

František Štampach

stampach@math.su.se

Boris Shapiro

shapiro@math.su.se

1 Department of Mathematics, Stockholm University, Kräftriket 5, 10691 Stockholm, Sweden 


\section{Introduction}

With a Laurent series

$$
a(z)=\sum_{k=-\infty}^{\infty} a_{k} z^{k}
$$

with complex coefficients $a_{k}$, one can associate a semi-infinite Toeplitz matrix $T(a)$ whose elements are given by

$$
(T(a))_{i, j}=a_{i-j}, \quad \forall i, j \in \mathbb{N}_{0}
$$

In the theory of Toeplitz matrices, $a$ is referred to as the symbol of $T(a)$ and the set of symbols under consideration may be further restricted depending on what properties of $T(a)$ are studied. For the spectral analysis of $T(a)$, a special role is played by the Wiener algebra which consists of symbols $a$ defined on the unit circle $\mathbb{T}$ whose Laurent series (1) is absolutely convergent for $z \in \mathbb{T}$. In this case, $T(a)$ is a bounded operator on the Banach space $\ell^{p}(\mathbb{N})$ for any $1 \leq p \leq \infty$, and its spectral properties are well known, see [5].

Rather than the actual spectrum of $T(a)$, this paper focuses on an asymptotic spectrum of $T(a)$, meaning the set of all limit points of eigenvalues of matrices $T_{n}(a)$, as $n \rightarrow \infty$, where $T_{n}(a)$ denotes the $n \times n$ principal submatrix of $T(a)$. An intimately related subject concerns the asymptotic eigenvalue distribution of Toeplitz matrices for which the most complete results were obtained if the symbol of the matrix is a Laurent polynomial [5]. More precisely, one considers symbols of the form

$$
b(z)=\sum_{k=-r}^{s} a_{k} z^{k}, \text { where } a_{-r} a_{s} \neq 0 \text { and } r, s \in \mathbb{N} \text {, }
$$

for which the Toeplitz matrix $T(b)$ is banded and is not lower or upper triangular.

This subject has an impressive history going back a century to the famous work of Szegő on the asymptotic behavior of the determinant of $T_{n}(b)$ for $n \rightarrow \infty$. The most essential progress was achieved by Schmidt and Spitzer [27] and later on by Hirschman [19]. They established the following results. The eigenvalue-counting measures

$$
\mu_{n}:=\frac{1}{n} \sum_{k=1}^{n} \delta_{\lambda_{k, n}}
$$

where $\lambda_{1, n}, \lambda_{2, n}, \ldots, \lambda_{n, n}$ are the eigenvalues of $T_{n}(b)$ (repeated according to their algebraic multiplicity) and $\delta_{a}$ is the Dirac measure supported at $\{a\}$, converge weakly to a limiting measure $\mu$, as $n \rightarrow \infty$. Recall that $\mu_{n}$ converges weakly to $\mu$, as $n \rightarrow \infty$, if and only if 


$$
\lim _{n \rightarrow \infty} \int_{\mathbb{C}} f(\lambda) \mathrm{d} \mu_{n}(\lambda)=\int_{\mathbb{C}} f(\lambda) \mathrm{d} \mu(\lambda),
$$

for every bounded and continuous function $f$ on $\mathbb{C}$. The limiting measure $\mu$ is supported on the limiting set

$$
\Lambda(b):=\left\{\lambda \in \mathbb{C} \mid \lim _{n \rightarrow \infty} \operatorname{dist}\left(\lambda, \operatorname{spec}\left(T_{n}(b)\right)\right)=0\right\} .
$$

The limit that appears above exists, see [27] or [5, Chp. 11]. Moreover, Schmidt and Spitzer derived another very useful description of $\Lambda(b)$ that reads

$$
\Lambda(b)=\left\{\lambda \in \mathbb{C}|| z_{r}(\lambda)|=| z_{r+1}(\lambda) \mid\right\},
$$

where $z_{1}(\lambda), z_{2}(\lambda), \ldots, z_{r+s}(\lambda)$ are (not necessarily distinct) roots of the polynomial $z \mapsto z^{r}(b(z)-\lambda)$ arranged in the order of increase of their absolute values, i.e.,

$$
0<\left|z_{1}(\lambda)\right| \leq\left|z_{2}(\lambda)\right| \leq \cdots \leq\left|z_{r+s}(\lambda)\right|
$$

(If there is a chain of equalities above, then the labeling of roots within this chain is arbitrary.)

The latter description allows one to deduce the fundamental analytical and topological properties of $\Lambda(b)$. First, the set $\Lambda(b)$ equals the union of a finite number of pairwise disjoint open analytic arcs and a finite number of the so-called exceptional points. A point $\lambda \in \Lambda(b)$ is called exceptional if either $\lambda$ is a branch point, i.e., if the polynomial $z \mapsto z^{r}(b(z)-\lambda)$ has a multiple root, or if there is no open neighborhood $U$ of $\lambda$ such that $\Lambda(b) \cap U$ is an analytic arc starting and terminating on the boundary $\partial U$. Second, $\Lambda(b)$ is a compact and connected set, see [34]. For an extension of these results to Toeplitz matrices with rational symbols, see the work of Day $[10,11]$.

As a starting point, we focus on a characterization of Laurent polynomial symbols $b$ for which $\Lambda(b) \subset \mathbb{R}$. In this case, we say that b belongs to the class $\mathscr{R}$; see Definition 2 . Besides all the symbols $b$ for which $T(b)$ is self-adjoint, the class $\mathscr{R}$ also contains symbols of certain non-self-adjoint matrices. Surprisingly, it turns out that, for $b \in \mathscr{R}$, the spectra of all submatrices $T_{n}(b)$ are real; see Theorem 1 below.

Describing the class $\mathscr{R}$ can be viewed as a problem of characterization of the subclass of banded Toeplitz matrices with asymptotically real spectrum. Let us point out that, in this context, the consideration of the set of the limit spectral points, i.e., $\Lambda(b)$, is a more relevant and interesting problem than the study of the spectrum of $T(b)$ as an operator acting on $\ell^{p}(\mathbb{N})$ for some $1 \leq p \leq \infty$. Indeed, note that $T(b)$ is self-adjoint if and only if $b(\mathbb{T}) \subset \mathbb{R}$, where $\mathbb{T}$ is the unit circle. On the other hand, $b(\mathbb{T})$ coincides with the essential spectrum of $T(b)$, see [5, Cor. 1.10]. Thus, if $T(b)$ is non-self-adjoint, then there is no chance for $\operatorname{spec}(T(b))$ to be purely real.

From a broader perspective, results of the present paper contribute to the study of the spectral properties of non-self-adjoint operators, a rapidly developing area which has recently attracted attention of many mathematicians and physicists, see, e.g., $[9,18$, 33]. A particularly noteworthy problem consists in finding classes of non-self-adjoint operators that have purely real spectrum. This is usually a mathematically challenging 
problem (see, for example, the proof of reality of the spectrum of the imaginary cubic oscillator in [28]) which, in addition, may be of physical relevance. At the moment, the non-self-adjoint operators whose spectrum is known to be real mainly comprise either very specific operators $[17,28,30,35]$ or operators that are in a certain sense close to being self-adjoint $[6,23,24]$. In particular, there are almost no criteria for famous non-self-adjoint families (such as Toeplitz, Jacobi, Hankel, Schrödinger, etc.) guaranteeing the reality of their spectra. From this point of view and to the best of our knowledge, the present article provides the first relevant results of such a flavor for the class of banded Toeplitz matrices.

Our main result is the following characterization of the class $\mathscr{R}$.

Theorem 1 Let b be a complex Laurent polynomial as in (2). The following statements are equivalent:

(i) $\Lambda(b) \subset \mathbb{R}$;

(ii) the set $b^{-1}(\mathbb{R})$ contains (an image of) a Jordan curve;

(iii) for all $n \in \mathbb{N}$, $\operatorname{spec}\left(T_{n}(b)\right) \subset \mathbb{R}$.

It is a very peculiar feature of banded Toeplitz matrices that the asymptotic reality of the eigenvalues (claim (i)) forces all eigenvalues of all principal submatrices to be real (claim (iii)). Hence, if, for instance, the $2 \times 2$ matrix $T_{2}(b)$ has a nonreal eigenvalue, there is no chance for the limiting set $\Lambda(b)$ to be real. The implication (iii) $\Rightarrow$ (i) is clearly trivial. The implication (i) $\Rightarrow$ (ii) is proved in Theorem 5 , while (ii) $\Rightarrow$ (iii) is established in Theorem 8 for an even more general class of symbols. These results are worked out within Sect. 2.

Section 3 is divided into two subsections. Section 3.1 is primarily devoted to a derivation of a new expression for the density of the limiting measure $\mu$ for real Laurent polynomial symbols $b \in \mathscr{R}$ using the polar parametrization of the Jordan curve from $b^{-1}(\mathbb{R})$ provided that such parametrization exists. Recall that due to Hirschman [19], a formula for the limiting density is known for any Laurent polynomial symbol (2) with no additional restrictions. The new expression, in the first place, emphasizes that the significance of the Jordan curve from $b^{-1}(\mathbb{R})$ does not concern only the reality questions as Theorem 1 shows, but it also provides enough data for a complete description of the limiting density. Secondly, the new formula does not depend directly on the zeros of $z \mapsto z^{r}(b(z)-\lambda)$, which might be more useful in practice. A more detailed comparison of the formulas for the limiting density is given in Remark 18.

The proof of the alternative description of the limiting measure $\mu$ uses the fact that $\mu$ coincides with a solution of a particular Hamburger moment problem whose moment sequence is determined by the symbol $b \in \mathscr{R}$. The positive-definiteness of the moment Hankel matrix then provides a necessary (algebraic) condition for $b$ to belong to $\mathscr{R}$. Whether the positive-definiteness of the Hankel matrices is also sufficient for $\Lambda(b) \subset \mathbb{R}$ remains an interesting open problem. It is only shown to be affirmative under an additional assumption requiring $\mathbb{C} \backslash \Lambda(b)$ to be a connected set, see the end of Sect. 3.1.

In Sect. 3.2, as a slight digression from the main topic, we briefly study the inverse spectral problem for self-adjoint Jacobi operators whose spectral measures coincide with the limiting measures for real $b \in \mathscr{R}$. By taking advantage of earlier results due 
to Hirschman and Rakhmanov, we prove that any such Jacobi operator is a compact perturbation of a Jacobi operator with constant diagonal and off-diagonal sequences. In the terminology of orthogonal polynomials, this means that any family of polynomials orthogonal with respect to the limiting measure, for real $b \in \mathscr{R}$, belongs to the Blumenthal-Nevai class.

Section 4 provides several concrete examples and numerical computations illustrating the results of Sects. 2 and 3. For general 3-diagonal and 4-diagonal Toeplitz matrices $T(b)$, explicit conditions in terms of $b$ guaranteeing that $b \in \mathscr{R}$ are deduced. Further, for the 3-diagonal and slightly specialized 4-diagonal Toeplitz matrices, the densities of the limiting measures as well as the associated Jacobi matrices are obtained fully explicitly. Moreover, we show that the corresponding orthogonal polynomials are related to certain well-known families of orthogonal polynomials, which involve Chebyshev polynomials of the second kind and the associated Jacobi polynomials. Their properties such as the three-term recurrence, the orthogonality relation, and an explicit representation are given. In the example concerning the 4-diagonal Toeplitz matrix, these results seem to be new. Yet for another interesting example generalizing the previous two, we are able to obtain some partial results. These examples are presented in Sects. 4.1-4.3. Finally, the last part of the paper contains various numerical illustrations and plots of the densities of the limiting measures and the distributions of eigenvalues in the situations whose complexity does not allow us to treat them explicitly.

\section{Main Results}

In this section, we prove Theorem 1.

Definition 2 Laurent polynomial $b$ of the form (2) is said to belong to the class $\mathscr{R}$, denoted by $b \in \mathscr{R}$, if and only if $\Lambda(b) \subset \mathbb{R}$, where $\Lambda(b)$ is given by (4).

Remark 3 Since $\Lambda(b)$ is a compact connected set, the inclusion $\Lambda(b) \subset \mathbb{R}$ actually implies that $\Lambda(b)$ is a closed finite interval.

We start with the proof of the implication (i) $\Rightarrow$ (ii) from Theorem 1 . For this purpose, we take a closer look at the structure of the set $b^{-1}(\mathbb{R})$ where the symbol is real-valued. Let us stress that $b^{-1}(\mathbb{R}) \subset \mathbb{C} \backslash\{0\}$. Recall also that a curve is a continuous mapping from a closed interval to a topological space and a Jordan curve is a curve which is, in addition, simple and closed. Occasionally, we will slightly abuse the term curve, sometimes meaning the mapping and sometimes the image of such a mapping. Below, a region always means an open and connected subset of $\mathbb{C}$.

Clearly, $z \in b^{-1}(\mathbb{R})$ if and only if $\operatorname{Im} b(z)=0$, and the latter condition can be turned into a polynomial equation $P(x, y)=0$, where $P \in \mathbb{R}[x, y], x=\operatorname{Re} z$, and $y=\operatorname{Im} z$. Consequently, $b^{-1}(\mathbb{R})$ is a finite union of pairwise disjoint open analytic arcs, i.e., images of analytic mappings from open intervals to $\mathbb{C}$, and a finite number of branching points that are the critical points of $b$. It might be convenient to add the points 0 and $\infty$ to $b^{-1}(\mathbb{R})$ and introduce the set

$$
n_{b}:=b^{-1}(\mathbb{R}) \cup\{0, \infty\}
$$


endowed with the topology induced from the Riemann sphere $S^{2}$. Then the set $n_{b}$ coincides with a set called net of the rational function $b$ by some authors, cf. [15].

As far as the local structure of $n_{b}$ at the points 0 and $\infty$ is concerned, notice that, since $b(z) \sim a_{-r} / z^{r}$, as $z \rightarrow 0, n_{b}$ looks locally at 0 like a star graph with $2 r$ edges and the angle between two consecutive edges is $\pi / r$. Similarly, since $b(z) \sim a_{s} z^{s}$, as $z \rightarrow \infty, n_{b}$ looks locally at $\infty$ like a star graph with $2 s$ edges with equal angles of magnitude $\pi / s$.

Concerning the set $n_{b}$ outside the singular points 0 and $\infty$, an important observation is that no arc of $n_{b}$ can terminate in $\mathbb{C} \backslash\{0\}$. This fact follows from Lemma 24 from the Appendix and the assumption that $b$ is a nonconstant Laurent polynomial. Consequently, any arc of $n_{b}$ located in $\mathbb{C} \backslash\{0\}$ has to be connected with 0 and/or $\infty$, or the arc closes into a loop entirely located in $\mathbb{C} \backslash\{0\}$. In the latter case, the loop gives rise to a Jordan curve that has to have the singular point 0 in its interior because, if the interior is a subset of $\mathbb{C} \backslash\{0\}$, then $b$ is an analytic function in the interior with real values on its boundary. This would, however, imply that $b$ is constant by Lemma 26 , a contradiction with our assumptions.

In total, the net $n_{b}$ is a collection of finitely many curves of 3 different types: Jordan curves containing either 0 or $\infty$, curves connecting 0 and $\infty$, and possibly Jordan curves entirely located in $\mathbb{C} \backslash\{0\}$ having 0 in the interior. To complete the picture, let us mention that, if $b^{-1}(\mathbb{R}) \subset \mathbb{C} \backslash\{0\}$ contains the Jordan curve, then it is unique. Indeed, assuming the opposite, i.e., the existence of two nonidentical Jordan curves in $b^{-1}(\mathbb{R})$ with 0 in their interiors, one can find a bounded nonempty region on which boundary $b$ would be real-valued. For this region, one can choose any connected component of the symmetric difference of the interiors of the two Jordan curves in question. By applying Lemma 26 again, one gets, however, that such $b$ has to be constant. In summary, the set $b^{-1}(\mathbb{R})$ either contains no Jordan curve or exactly one. The two possibilities for the set $b^{-1}(\mathbb{R})$ that are to be distinguished are illustrated in Fig. 1.

With the above information about the structure of the net $n_{b}$, the verification of the following lemma is immediate.

Lemma 4 The set $b^{-1}(\mathbb{R})$ contains a Jordan curve if and only if every path in $S^{2}$ connecting 0 and $\infty$ has a nonempty intersection with $b^{-1}(\mathbb{R})$.

Now, we are ready to show that the existence of a Jordan curve in $b^{-1}(\mathbb{R})$ is necessary for $\Lambda(b) \subset \mathbb{R}$, i.e., the implication (i) $\Rightarrow$ (ii) from Theorem 1 .

Theorem 5 If $\Lambda(b) \subset \mathbb{R}$, then $b^{-1}(\mathbb{R})$ contains a Jordan curve.

Proof Set

$$
\mathcal{N}:=\left\{z \in \mathbb{C}|| z_{r}(b(z))|=| z_{r+1}(b(z)) \mid\right\} .
$$

Note that $b$ maps a neighborhood of 0 and a neighborhood of $\infty$ onto a neighborhood of $\infty$ and that for $\lambda$ large in the modulus, the preimages $z_{1}(\lambda), \ldots, z_{r}(\lambda)$ are close to 0 , while $z_{r+1}(\lambda), \ldots, z_{r+s}(\lambda)$ are close to $\infty$. Hence there exist neighborhoods of 0 and $\infty$ with empty intersections with $\mathcal{N}$. 

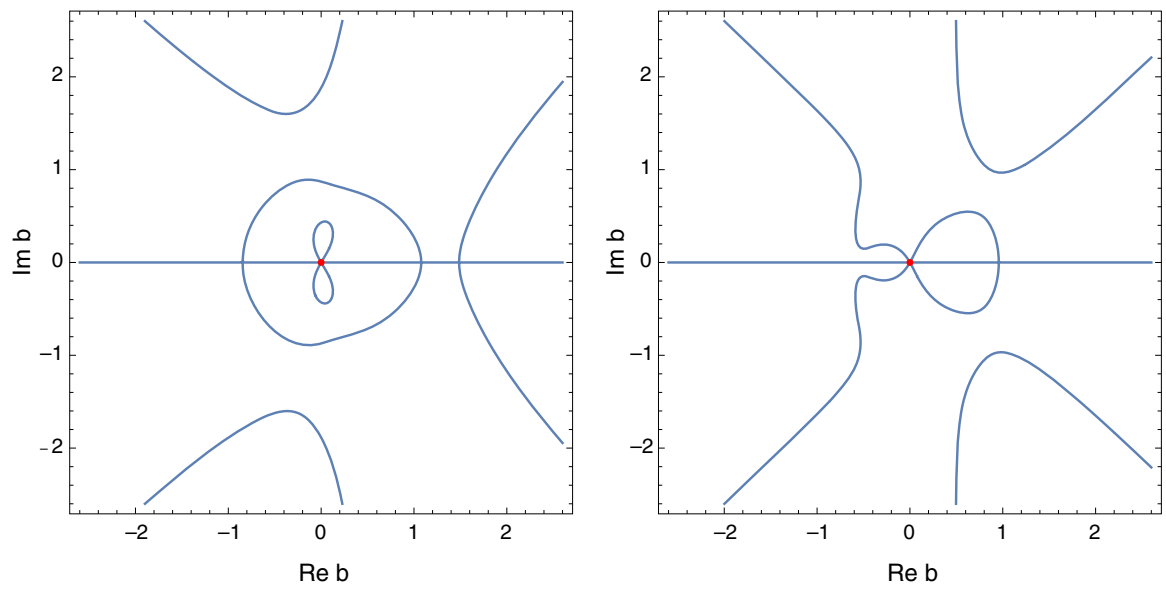

Fig. 1 The plots of the net of $b(z)=1 / z^{3}-1 / z^{2}+7 / z+9 z-2 z^{2}+2 z^{3}-z^{4}$ (left) and $b(z)=$ $-2 / z^{3}-4 / z^{2}+12 / z+8 z-10 z^{2}+8 z^{3}-4 z^{4}$ (right). The red dot designates the origin. On the left-hand side, $b^{-1}(\mathbb{R})$ contains a Jordan curve, while this is not the case for the example plotted on the right-hand side (Color figure online)

Next, let us show that any path in $S^{2}$ connecting 0 and $\infty$ has a nonempty intersection with $\mathcal{N}$. Let $\gamma:[0,1] \rightarrow S^{2}$ denote such a path. First, note that, for any $k \in\{1, \ldots, r+$ $s\}$, the function $\lambda \mapsto\left|z_{k}(\lambda)\right|$ is continuous on $\mathbb{C} \backslash\{0\}$ and that the value $|\gamma(t)|$ appears at least once in the $(r+s)$-tuple $\left|z_{1}(b(\gamma(t)))\right|, \ldots,\left|z_{r+s}(b(\gamma(t)))\right|$ for all $t \in(0,1)$. Further, since $\gamma(0)=0$, the value $|\gamma(t)|$, for $t$ in a right neighborhood of 0 , appears among the first $r$ values $\left|z_{1}(b(\gamma(t)))\right|, \ldots,\left|z_{r}(b(\gamma(t)))\right|$ and does not appear in the remaining $s$ values $\left|z_{r+1}(b(\gamma(t)))\right|, \ldots,\left|z_{r+s}(b(\gamma(t)))\right|$. Similarly, since $\gamma(1)=\infty$, the value $|\gamma(t)|$, for $t$ in a left neighborhood of 1 , appears in $\left|z_{r+1}(b(\gamma(t)))\right|, \ldots,\left|z_{r+s}(b(\gamma(t)))\right|$ and is not among $\left|z_{1}(b(\gamma(t)))\right|, \ldots,\left|z_{r}(b(\gamma(t)))\right|$. Since $\gamma$ is a continuous map, there must exist $t_{0} \in(0,1)$ such that $\left|\gamma\left(t_{0}\right)\right|=\left|z_{r}\left(b\left(\gamma\left(t_{0}\right)\right)\right)\right|=\left|z_{r+1}\left(b\left(\gamma\left(t_{0}\right)\right)\right)\right|$. Hence $\gamma((0,1)) \cap \mathcal{N} \neq \varnothing$.

Finally, recalling (4), one has $b(\mathcal{N}) \subset \Lambda(b) \subset \mathbb{R}$, hence $\mathcal{N} \subset b^{-1}(\mathbb{R})$. Consequently, we obtain that any path in $S^{2}$ joining 0 and $\infty$ has a nonempty intersection with $b^{-1}(\mathbb{R})$ and Theorem 5 follows from Lemma 4.

The second part of this subsection is devoted to the proof of the implication (ii) $\Rightarrow$ (iii) of Theorem 1 . Recall that every Jordan curve in $\mathbb{C}$ is homeomorphic to the unit circle $\mathbb{T}:=\{z \in \mathbb{C}|| z \mid=1\}$ and therefore it gives rise to a homeomorphic mapping $\gamma: \mathbb{T} \rightarrow \mathbb{C}$. In addition, the unit circle is naturally parametrized by the polar angle $t \mapsto e^{\mathrm{i} t}$, where $t \in[-\pi, \pi]$. In the following, we always choose the parametrization of a Jordan curve $\gamma=\gamma(t)$, with $t \in[-\pi, \pi]$, which can be viewed as the composition of the above two mappings.

Note that the mapping $z \rightarrow 1 / \bar{z}$ reflects the points in $\mathbb{C} \backslash\{0\}$ with respect to $\mathbb{T}$. For a given Jordan curve $\gamma$ with 0 in its interior, we define a new Jordan curve $\gamma^{*}$ by reflecting $\gamma$ with respect to $\mathbb{T}$, i.e., $\gamma^{*}:=1 / \bar{\gamma}$. In other words, if $\gamma$ is parametrized as

$$
\gamma(t)=\rho(t) e^{\mathrm{i} \phi(t)}
$$


with a positive function $\rho$ and real function $\phi$, then

$$
\gamma^{*}(t)=\frac{1}{\rho(t)} e^{\mathrm{i} \phi(t)} .
$$

We also introduce the notation

$$
r_{\gamma}:=\min _{t \in[-\pi, \pi]}|\gamma(t)| \text { and } R_{\gamma}:=\max _{t \in[-\pi, \pi]}|\gamma(t)|
$$

In the following lemma, $\langle\cdot, \cdot\rangle$ stands for the standard inner product on $\mathbb{C}^{n}$; further, with any vector $u=\left(u_{0}, u_{1}, \ldots, u_{n-1}\right) \in \mathbb{C}^{n}$, we associate the polynomial $f_{u}(z):=$ $u_{0}+u_{1} z+\cdots+u_{n-1} z^{n-1}$.

Lemma 6 Let $\gamma$ be a Jordan curve with 0 in its interior and a be a function given by the Laurent series

$$
a(z)=\sum_{k=-\infty}^{\infty} a_{k} z^{k}
$$

which is absolutely convergent in the annulus $r_{\gamma} \leq|z| \leq R_{\gamma}$. Then, for all $u, v \in \mathbb{C}^{n}$ and $n \in \mathbb{N}$, one has

$$
\left\langle u, T_{n}(a) v\right\rangle=\left\langle f_{u}, a f_{v}\right\rangle_{\gamma}:=\frac{1}{2 \pi \mathrm{i}} \int_{-\pi}^{\pi} a(\gamma(t)) f_{v}(\gamma(t)) \overline{f_{u}\left(\gamma^{*}(t)\right)} \frac{\dot{\gamma}(t)}{\gamma(t)} \mathrm{d} t .
$$

Proof The proof proceeds by a straightforward computation. We have

$$
\begin{aligned}
\left\langle f_{u}, a f_{v}\right\rangle_{\gamma} & =\frac{1}{2 \pi \mathrm{i}} \int_{-\pi}^{\pi} a(\gamma(t)) f_{v}(\gamma(t)) \overline{f_{u}\left(\gamma^{*}(t)\right)} \frac{\dot{\gamma}(t)}{\gamma(t)} \mathrm{d} t \\
& =\frac{1}{2 \pi \mathrm{i}} \sum_{k=-\infty}^{\infty} \sum_{l=0}^{n-1} \sum_{m=0}^{n-1} a_{k} v_{l} \overline{u_{m}} \int_{-\pi}^{\pi}(\gamma(t))^{k+l-m-1} \dot{\gamma}(t) \mathrm{d} t \\
& =\sum_{l=0}^{n-1} \sum_{m=0}^{n-1} a_{m-l} v_{l} \overline{u_{m}}=\left\langle u, T_{n}(a) v\right\rangle,
\end{aligned}
$$

where we have used that

$$
\frac{1}{2 \pi \mathrm{i}} \int_{-\pi}^{\pi}(\gamma(t))^{j-1} \dot{\gamma}(t) \mathrm{d} t=\frac{1}{2 \pi \mathrm{i}} \oint_{\gamma} z^{j-1} \mathrm{~d} z=\delta_{j, 0}
$$

for any $j \in \mathbb{Z}$.

Corollary 7 For any element a of the Wiener algebra, $u, v \in \mathbb{C}^{n}$, and $n \in \mathbb{N}$, one has

$$
\left\langle u, T_{n}(a) v\right\rangle=\left\langle f_{u}, a f_{v}\right\rangle_{\mathbb{T}}:=\frac{1}{2 \pi} \int_{-\pi}^{\pi} a\left(e^{\mathrm{i} t}\right) f_{v}\left(e^{\mathrm{i} t}\right) \overline{f_{u}\left(e^{\mathrm{i} t}\right)} \mathrm{d} t .
$$


Proof Choose $\gamma(t)=e^{\mathrm{i} t}, t \in[-\pi, \pi]$, in Lemma 6.

Theorem 8 Let $\gamma$ be a Jordan curve with 0 in its interior and a be a function given by the Laurent series

$$
a(z)=\sum_{k=-\infty}^{\infty} a_{k} z^{k}
$$

which is absolutely convergent in the annulus $r_{\gamma} \leq|z| \leq R_{\gamma}$. Suppose further that $a(\gamma(t)) \in \mathbb{R}$ for all $t \in[-\pi, \pi]$. Then

$$
\operatorname{spec}\left(T_{n}(a)\right) \subset \mathbb{R}, \quad \forall n \in \mathbb{N}
$$

Remark 9 In particular, if the Laurent series of the symbol $a$ from Theorem 8 converges absolutely for all $z \in \mathbb{C} \backslash\{0\}$, then $\operatorname{spec}\left(T_{n}(a)\right) \subset \mathbb{R}$ for all $n \in \mathbb{N}$, provided that $a^{-1}(\mathbb{R})$ contains a Jordan curve (which has to contain 0 in its interior). This applies for Laurent polynomial symbols (2) especially, which yields the implication (ii) $\Rightarrow$ (iii) of Theorem 1.

Another family of Toeplitz matrices, for which Theorem 8 is readily applicable, are those with rational symbols studied by Day in $[10,11]$. Using the notation of the definition from [11, Sec. 1], the rational symbol $f$ has the absolutely convergent Laurent series expansion in the annulus given by inequalities $R_{1}<|z|<R_{2}$. If this annulus contains a Jordan curve on which $f$ has real values, all eigenvalues of $T_{n}(f)$ are real for an arbitrary $n \in \mathbb{N}$.

Proof of Theorem 8 First note that we can assume that $\mathbb{T} \subset\left\{z \in \mathbb{C}\left|r_{\gamma} \leq\right| z \mid \leq R_{\gamma}\right\}$ without loss of generality. Indeed, if this is not the case, we consider the symbol $a_{\rho}(z):=a(\rho z)$, where $\rho>0$ is such that $r_{\gamma} \leq \rho \leq R_{\gamma}$. Then the Laurent series expansion of $a_{\rho}$ is absolutely convergent in the annulus $r_{\gamma} / \rho \leq|z| \leq$ $R_{\gamma} / \rho$ which contains $\mathbb{T}$ and $a_{\rho}$ has real values on the Jordan curve $\gamma / \rho$. Moreover, $\operatorname{spec}\left(T_{n}(a)\right)=\operatorname{spec}\left(T_{n}\left(a_{\rho}\right)\right)$ for all $n \in \mathbb{N}$, because the matrices $T_{n}(a)$ and $T_{n}\left(a_{\rho}\right)$ are similar via the similarity relation $T_{n}\left(a_{\rho}\right)=D_{n}(\rho) T_{n}(a) D_{n}^{-1}(\rho)$, where $D_{n}(\rho)=\operatorname{diag}\left(1, \rho, \rho^{2}, \ldots, \rho^{n-1}\right)$.

Let $\mu \in \operatorname{spec}\left(T_{n}(a)\right)$ and $u \in \mathbb{C}^{n}$ be the normalized eigenvector corresponding to $\mu$. Then, by using Corollary 7 , one obtains

$$
\mu=\left\langle u, T_{n}(a) u\right\rangle=\left\langle f_{u}, a f_{u}\right\rangle_{\mathbb{T}}=\overline{\left\langle f_{u}, \bar{a} f_{u}\right\rangle_{\mathbb{T}}}
$$

On the other hand, by applying Lemma 6 twice, one gets

$$
\left\langle f_{u}, \bar{a} f_{u}\right\rangle_{\mathbb{T}}=\left\langle u, T_{n}(\bar{a}) u\right\rangle=\left\langle f_{u}, \bar{a} f_{u}\right\rangle_{\gamma}=\left\langle f_{u}, a f_{u}\right\rangle_{\gamma}
$$

where the last equality holds since $a(\gamma(t)) \in \mathbb{R}$ for all $t \in[-\pi, \pi]$, by the assumption. Finally, by using (6) together with (7) and applying Lemma 6 again, one arrives at the equality

$$
\mu=\overline{\left\langle f_{u}, a f_{u}\right\rangle_{\gamma}}=\overline{\left\langle u, T_{n}(a) u\right\rangle}=\bar{\mu}
$$


Hence $\mu \in \mathbb{R}$.

Remark 10 Note that the entries of the Toeplitz matrix considered in Theorem 8 are allowed to be complex. Clearly, there exists Toeplitz matrices satisfying assumptions of Theorem 8 with nonreal entries, for instance, any self-adjoint Toeplitz matrix with nonreal entries whose symbol belongs to the Wiener algebra. On the other hand, if a Toeplitz matrix is at the same time an upper or lower Hessenberg matrix, then its entries have to be real. More precisely, if the symbol has the form

$$
a(z)=\frac{1}{z}+\sum_{n=0}^{\infty} a_{n} z^{n}
$$

and the assumptions of Theorem 8 are fulfilled, then $a_{n} \in \mathbb{R}$ for all $n \in \mathbb{N}_{0}$. Indeed, it is easy to verify that $D_{n}:=\operatorname{det} T_{n}(a)$ satisfies the recurrence

$$
D_{n}=(-1)^{n-1} a_{n-1}+\sum_{k=0}^{n-2}(-1)^{k} a_{k} D_{n-k-1}, \quad \forall n \in \mathbb{N}
$$

Since, by Theorem 8, all eigenvalues of $T_{n}(a)$ are real, $D_{n} \in \mathbb{R}$ for any $n \in \mathbb{N}$. Hence one can use that $a_{0}=D_{1} \in \mathbb{R}$ and the above recurrence to prove by induction that $a_{n} \in \mathbb{R}$ for all $n \in \mathbb{N}_{0}$.

\section{Formula for the Limiting Density and Other Results}

For the analysis of this subsection, we restrict ourself with real banded Toeplitz matrices $T(b)$. More precisely, we consider symbols

$$
b(z)=\sum_{k=-r}^{s} a_{k} z^{k}, \text { where } a_{k} \in \mathbb{R}, a_{-r} a_{s} \neq 0 \text { and } r, s \in \mathbb{N} .
$$

\subsection{The Limiting Density and the Hamburger Moment Problem}

Our first goal is to provide a more detailed description of the limiting measure $\mu$ for the symbols (8) in terms of the Jordan curve $\gamma$ contained in $b^{-1}(\mathbb{R})$. For the sake of simplicity, we focus on the situation when the Jordan curve admits the polar parametrization

$$
\gamma(t)=\rho(t) e^{\mathrm{i} t}, \quad t \in[-\pi, \pi]
$$

where $\rho(t)>0$ for all $t \in[-\pi, \pi]$. We should mention that so far, we have not observed any example of $b \in \mathscr{R}$ where the Jordan curve in $b^{-1}(\mathbb{R})$ would intersect a radial ray at more than one point, in which case the parametrization (9) would be impossible for such a curve. In particular, all the examples presented in Sect. 4 where $\gamma$ is known explicitly satisfy (9). 
Incidentally, the problem of description of the limiting measure for $b \in \mathscr{R}$ is closely related to the classical Hamburger moment problem. Standard references on this subject are $[2,7,29,31]$. A solution of the Hamburger moment problem with a moment sequence $\left\{h_{m}\right\}_{m \in \mathbb{N}_{0}} \subset \mathbb{R}, h_{0}=1$, is a probability measure $\mu$ supported in $\mathbb{R}$ with all moments finite and equal to $h_{m}$, i.e.,

$$
\int_{\mathbb{R}} x^{m} \mathrm{~d} \mu(x)=h_{m}, \quad \forall m \in \mathbb{N}_{0} .
$$

By the well-known Hamburger theorem, the Hamburger moment problem with a moment sequence $\left\{h_{m}\right\}_{m \in \mathbb{N}_{0}}, h_{0}=1$, has a solution if and only if the Hankel matrix

$$
H_{n}:=\left(\begin{array}{cccc}
h_{0} & h_{1} & \ldots & h_{n-1} \\
h_{1} & h_{2} & \ldots & h_{n} \\
\vdots & \vdots & \ddots & \vdots \\
h_{n-1} & h_{n} & \ldots & h_{2 n-2}
\end{array}\right)
$$

is positive definite for all $n \in \mathbb{N}$. The solution may be unique (the determinate case) or they can be infinitely many (the indeterminate case). However, if there is a solution whose support is compact, then it is unique, see, for example, [31, Prop. 1.5].

For a later purpose, let us also recall the formula

$$
\lim _{n \rightarrow \infty} \frac{1}{n} \operatorname{Tr}\left(T_{n}(b)\right)^{m}=\frac{1}{2 \pi} \int_{-\pi}^{\pi} b^{m}\left(e^{\mathrm{i} t}\right) \mathrm{d} t, \quad m \in \mathbb{N},
$$

which is usually attributed to G. Szegó. In fact, Szegó proved (12) for any symbol $b$ belonging to the Wiener class, imposing however, an additional assumption that is equivalent to the self-adjointness of $T(b)$. Later on, M. Kac showed that (12) remains valid for any $b$ from the Wiener class without the additional assumption, see [20].

Lemma 11 Let $b \in \mathscr{R}$ have the form (8). Then the Hamburger moment problem with the moment sequence given by

$$
h_{0}:=1 \text { and } h_{m}:=\frac{1}{2 \pi} \int_{-\pi}^{\pi} b^{m}\left(e^{\mathrm{i} t}\right) \mathrm{d} t, \quad m \in \mathbb{N},
$$

has a solution that is unique and coincides with the limiting measure $\mu$.

Proof First, note that $h_{m} \in \mathbb{R}$ for all $m \in \mathbb{N}_{0}$, since the coefficients of $b$ are assumed to be real. Moreover, since $b \in \mathscr{R}, \operatorname{supp} \mu \equiv \Lambda(b) \subset \mathbb{R}$ and it is a compact set. Hence, by the above discussion, it suffices to verify that $\mu$ satisfies (10). To this end, recall that $\mu$ is the weak limit of the measures $\mu_{n}$ given by (3). Then, by using the limit formula (12), one gets

$$
\int_{\mathbb{R}} x^{m} \mathrm{~d} \mu(x)=\lim _{n \rightarrow \infty} \int_{\mathbb{R}} x^{m} \mathrm{~d} \mu_{n}(x)=\lim _{n \rightarrow \infty} \frac{1}{n} \operatorname{Tr}\left(T_{n}(b)\right)^{m}=h_{m}
$$

for all $m \in \mathbb{N}_{0}$. 
Corollary 12 If $b \in \mathscr{R}$ is of the form (8), then the Hankel matrix (11) with entries given by (13) is positive definite for all $n \in \mathbb{N}$.

Note that if the coefficients of $b \in \mathscr{R}$ are real, then $\mathbb{R} \backslash\{0\} \subset b^{-1}(\mathbb{R})$ and $b^{-1}(\mathbb{R})$ is symmetric with respect to the real line. The Jordan curve $\gamma$ contained in $b^{-1}(\mathbb{R})$ intersects the real line at exactly two points (negative and positive) which are the critical points of $b$.

Theorem 13 Suppose that $b \in \mathscr{R}$ is as in (8) and the Jordan curve $\gamma$ contained in $b^{-1}(\mathbb{R})$ admits the polar parametrization (9). Further, let $\ell \in \mathbb{N}_{0}$ be the number of critical points of $b$ in $\gamma((0, \pi))$ and $0=: \phi_{0}<\phi_{1}<\cdots<\phi_{\ell}<\phi_{\ell+1}:=\pi$ be such that $b^{\prime}\left(\gamma\left(\phi_{j}\right)\right)=0$ for all $j \in\{0,1, \ldots, \ell+1\}$. Then $b \circ \gamma$ restricted to $\left(\phi_{i-1}, \phi_{i}\right)$ is strictly monotone for all $i \in\{1,2, \ldots, \ell+1\}$, and the limiting measure $\mu=\mu_{1}+\mu_{2}+\cdots+\mu_{\ell+1}$, where $\mu_{i}$ is an absolutely continuous measure supported on $\left[\alpha_{i}, \beta_{i}\right]:=b\left(\gamma\left(\left[\phi_{i-1}, \phi_{i}\right]\right)\right)$ whose density is given by

$$
\frac{\mathrm{d} \mu_{i}}{\mathrm{~d} x}(x)= \pm \frac{1}{\pi} \frac{\mathrm{d}}{\mathrm{d} x}(b \circ \gamma)^{-1}(x)
$$

for all $x \in\left(\alpha_{i}, \beta_{i}\right)$ and all $i \in\{1,2, \ldots, \ell+1\}$. In (14) the + sign is used when $b \circ \gamma$ increases on $\left(\alpha_{i}, \beta_{i}\right)$, and the - sign is used otherwise.

Remark 14 Notice that $\operatorname{supp} \mu=\left[\min _{1 \leq i \leq \ell+1} \alpha_{i}, \max _{1 \leq i \leq \ell+1} \beta_{i}\right]$. Further, observe that, in particular, Theorem 13 provides a description of the limiting measure for all self-adjoint real banded Toeplitz matrices since, in this case, the Jordan curve is just the unit circle. An illustration of this situation is given in Example 5 in Sect.4.5.

Proof of Theorem 13 First, note that, for any $i \in\{1,2 \ldots, \ell+1\}$, the first derivative of the smooth function $b \circ \gamma:\left(\phi_{i-1}, \phi_{i}\right) \rightarrow \mathbb{R}$ does not change sign on $\left(\phi_{i-1}, \phi_{i}\right)$ because

$$
(b \circ \gamma)^{\prime}(t)=b^{\prime}(\gamma(t))\left(\rho^{\prime}(t)+\mathrm{i} \rho(t)\right) e^{\mathrm{i} t}=0
$$

if and only if $t=\phi_{j}$ for some $j \in\{0,1, \ldots, \ell+1\}$. Hence $b \circ \gamma$ restricted to $\left(\phi_{i-1}, \phi_{i}\right)$ is either strictly increasing or strictly decreasing, and the same holds for the inverse $(b \circ \gamma)^{-1}:\left(\alpha_{i}, \beta_{i}\right) \rightarrow\left(\phi_{i-1}, \phi_{i}\right)$. Consequently, by formula (14), a positive measure $\mu_{i}$ supported on $\left[\alpha_{i}, \beta_{i}\right]$ is well defined for all $i \in\{1,2 \ldots, \ell+1\}$. Let us write $v:=\mu_{1}+\mu_{2}+\cdots+\mu_{\ell+1}$.

In the second part of the proof, we verify that the measure $v$ which is supported on $\left[\min _{1 \leq i \leq \ell+1} \alpha_{i}, \max _{1 \leq i \leq \ell+1} \beta_{i}\right]$ has the same moments (13) as the limiting measure $\mu$. Then $\mu=v$ by Lemma 11 .

Let $m \in \mathbb{N}_{0}$ be fixed. By deforming the positively oriented unit circle into the Jordan curve $\gamma$, one gets the equality

$$
h_{m}=\frac{1}{2 \pi \mathrm{i}} \oint_{\mathbb{T}} b^{m}(z) \frac{\mathrm{d} z}{z}=\frac{1}{2 \pi \mathrm{i}} \oint_{\gamma} b^{m}(z) \frac{\mathrm{d} z}{z} .
$$


Next, by using the symmetry $\overline{b(z)}=b(\bar{z})$ for $z \in \mathbb{C} \backslash\{0\}$, parametrization (9), and the fact that $b \circ \gamma$ is real-valued, one arrives at the expression

$$
h_{m}=\frac{1}{\pi} \int_{0}^{\pi} b^{m}(\gamma(t)) \mathrm{d} t .
$$

By splitting the above integral according to the positions of the critical points of $b$ on the arc of $\gamma$ in the upper half-plane, one further obtains

$$
h_{m}=\frac{1}{\pi} \sum_{i=1}^{\ell+1} \int_{\phi_{i-1}}^{\phi_{i}} b^{m}(\gamma(t)) \mathrm{d} t .
$$

Finally, since $b \circ \gamma$ restricted to $\left(\phi_{i-1}, \phi_{i}\right)$ is monotone, we can change the variable $x=b(\gamma(t))$ in each of the above integrals, getting

$$
h_{m}=\sum_{i=1}^{\ell+1} \int_{\alpha_{i}}^{\beta_{i}} x^{m} \mathrm{~d} \mu_{i}(x)=\int_{\mathbb{R}} x^{m} \mathrm{~d} v(x),
$$

which concludes the proof.

Under the assumptions of Theorem 13, the Cauchy transform of the measure $\mu$ can be expressed with the aid of the particular Jordan curve $\gamma$ from $b^{-1}(\mathbb{R})$.

Corollary 15 Suppose that $b \in \mathscr{R}$ has the form (8) and the Jordan curve $\gamma$ contained in $b^{-1}(\mathbb{R})$ is parametrized as in (9). Then, for all $z \notin \Lambda(b)$,

$$
\int_{\Lambda(b)} \frac{\mathrm{d} \mu(x)}{x-z}=\frac{1}{\pi} \int_{0}^{\pi} \frac{\mathrm{d} t}{b(\gamma(t))-z} .
$$

Proof Since $\Lambda(b)$ is a bounded interval, the Cauchy transform of $\mu$ can be expanded for $z$ sufficiently large in modulus as

$$
\int_{\Lambda(b)} \frac{\mathrm{d} \mu(x)}{x-z}=-\sum_{m=0}^{\infty} \frac{h_{m}}{z^{m+1}},
$$

where $h_{m}$ are the moments of $\mu$ as in (10). By plugging the expression (15) for $h_{m}$ into the above formula and proceeding similarly as before, one arrives at the identity (16) which is therefore verified for all $z$ from a neighborhood of $\infty$. Finally, by Theorem 13 , the interval $\Lambda(b)$ equals $b(\gamma[0, \pi])$, and hence both sides of (16) are analytic in $z$ on $\mathbb{C} \backslash \Lambda(b)$. The identity principle for analytic functions extends the validity of (16) to all $z \in \mathbb{C} \backslash \Lambda(b)$.

Generically, there is no critical point of $b$ located on the curve $\gamma$ in the upper halfplane, i.e., $\ell=0$ in Theorem 13 . Hence the only critical points located on the curve $\gamma$ are the two intersection points of $\gamma$ and the real line. In this case, the statement of Theorem 13 gets a simpler form. Although it is just a particular case of Theorem 13, we formulate this simpler statement separately below. 
Theorem 16 Suppose that $b \in \mathscr{R}$ is as in (8) and the Jordan curve $\gamma$ contained in $b^{-1}(\mathbb{R})$ admits the polar parametrization (9). Moreover, let $b^{\prime}(\gamma(t)) \neq 0$ for all $t \in(0, \pi)$. Then $b \circ \gamma$ restricted to $(0, \pi)$ is either strictly increasing or decreasing; the limiting measure $\mu$ is supported on the interval $[\alpha, \beta]:=b(\gamma([0, \pi]))$ and its density satisfies

$$
\frac{\mathrm{d} \mu}{\mathrm{d} x}(x)= \pm \frac{1}{\pi} \frac{\mathrm{d}}{\mathrm{d} x}(b \circ \gamma)^{-1}(x)
$$

for $x \in(\alpha, \beta)$, where the + sign is used when $b \circ \gamma$ increases on $(0, \pi)$ and the sign is used otherwise.

Remark 17 Since $b \circ \gamma$ is a one-to-one mapping from $[0, \pi]$ onto $[\alpha, \beta]$ under the assumptions of Theorem 16, we can use it for a reparametrization of the arc of $\gamma$ in the upper half-plane. Namely, we denote by $\gamma_{b}:=\gamma \circ(b \circ \gamma)^{-1}:[\alpha, \beta] \rightarrow\{z \in$ $\mathbb{C} \mid \operatorname{Im} z \geq 0\}$ the new parametrization of the arc of $\gamma$ to distinguish the notation. Recalling (9), one observes that

$$
\operatorname{Arg} \gamma_{b}(x)=(b \circ \gamma)^{-1}(x), \quad \forall x \in[\alpha, \beta]
$$

Then the limiting measure is determined by the change of the argument of the Jordan curve contained in $b^{-1}(\mathbb{R})$ provided that the parametrization $\gamma_{b}$ is used. More concretely, if we denote the distribution function of $\mu$ by $F_{\mu}:=\mu([\alpha, \cdot))$, then formula (17) can be rewritten as

$$
F_{\mu}(x)= \begin{cases}\frac{1}{\pi} \operatorname{Arg} \gamma_{b}(x) & \text { if } \gamma_{b} \text { is positively (counterclockwise) oriented } \\ 1-\frac{1}{\pi} \operatorname{Arg} \gamma_{b}(x) & \text { if } \gamma_{b} \text { is negatively (clockwise) oriented. }\end{cases}
$$

Yet another equivalent formulation reads

$$
F_{\mu}(b(\gamma(t)))= \begin{cases}\frac{1}{\pi} t & \text { if } b(\gamma(0))<b(\gamma(\pi)) \\ 1-\frac{1}{\pi} t & \text { if } b(\gamma(\pi))<b(\gamma(0))\end{cases}
$$

for $t \in[0, \pi]$

Remark 18 Let us point out certain aspects of the formulas appearing in Theorems 13, 16, and Remark 17 as compared to the general formula for the limiting density that is the main result of Hirschman's paper [19]. Recall that Hirschman's formula holds for an arbitrary Laurent polynomial symbol of the form (2). To write it down, it is convenient to introduce the following notation. Let $\Gamma$ denote an analytic arc from the representation of $\Lambda(b)$ mentioned in the Introduction of this paper, and set

$$
G_{1}(\lambda):=a_{s} \prod_{j=1}^{r}\left(-z_{j}(\lambda)\right) \quad \text { and } \quad G_{2}(\lambda):=a_{s} \prod_{j=r+1}^{r+s}\left(-z_{j}(\lambda)\right)
$$


where $z_{1}(\lambda), \ldots, z_{r+s}(\lambda)$ are the ordered zeros as in (5). The functions $G_{1}$ and $G_{2}$ are analytic in a neighborhood of $\Gamma$ and coincide in modulus on $\Gamma$. If $\lambda=\lambda(s)$ is the arc length parametrization of $\Gamma$, then Hirschman's formula reads

$$
\frac{\mathrm{d} \mu}{\mathrm{d} s}(s)=\frac{1}{2 \pi}\left|\frac{\mathrm{d}}{\mathrm{d} \lambda} \frac{G_{2}(\lambda)}{G_{1}(\lambda)}\right|_{\lambda=\lambda(s)} .
$$

Doing this for each arc $\Gamma$ of $\Lambda(b)$, one obtains the limiting density. A slightly different expression can also be found in [5, Thm. 11.16].

Yet another expression for the limiting density, which is equivalent to Hirschman's formula and appears in [13, Eq. (1.12)], reads

$$
\frac{\mathrm{d} \mu}{\mathrm{d} \lambda}(\lambda)=\frac{1}{2 \pi \mathrm{i}} \sum_{j=1}^{r}\left(\frac{z_{j+}^{\prime}(\lambda)}{z_{j+}(\lambda)}-\frac{z_{j-}^{\prime}(\lambda)}{z_{j-}(\lambda)}\right)
$$

Here $\mathrm{d} \lambda$ is the complex line element on $\Gamma$ taken with respect to a chosen orientation on $\Gamma$ and $z_{j \pm}(\lambda)$ are one-side limits of $z_{j}\left(\lambda^{\prime}\right)$, as $\lambda^{\prime}$ approaches $\lambda \in \Gamma$ from the left/right side of $\Gamma$ determined by the chosen orientation. In contrast to formula (19), it is not clear from (20) whether $\mu$ is positive. The complex expression (20) is more suitable for the analysis leading to the main result of [13] which shows that the limiting measure $\mu$ is in fact one component of a vector of measures minimizing a certain energy functional. Formulas for densities of the remaining measures similar to (20) are given in [13, Thm. 2.3].

Both formulas (19) and (20) hold for any $b$ of the form (2), while the validity of formula (14) is restricted by the assumptions of Theorem 13 when, in particular, $\operatorname{supp} \mu \subset \mathbb{R}$. The theoretical significance of Theorem 13 is that it demonstrates that the role of the special Jordan curve $\gamma$ on which $b$ has real values is essential not only for the set $\Lambda(b)$, where the eigenvalues of $T_{n}(b)$ cluster, but $\gamma$ also encodes with what density the eigenvalues accumulate on $\Lambda(b)$. In addition, the general formulas (19) and (20) depend on the roots $z_{1}(\lambda), \ldots, z_{r+s}(\lambda)$ while the alternative expressions from Theorems 13, 16, and Remark 17 require the knowledge of a parametrization of the Jordan curve from $b^{-1}(\mathbb{R})$ instead. This might be an advantage in some situations; see Sect. 4.

Let us once more go back to the Hamburger moment problem with the moment sequence given by (13). We derive an integral formula for det $H_{n}$.

Theorem 19 Let $b$ be as in (2) and $H_{n}$ be the Hankel matrix (11) with the entries given by (13). Then, for all $n \in \mathbb{N}$, one has

$$
\operatorname{det} H_{n}=\frac{1}{(2 \pi)^{n} n !} \int_{\pi}^{\pi} \int_{-\pi}^{\pi} \ldots \int_{-\pi}^{\pi} \prod_{1 \leq i<j \leq n}\left(b\left(e^{\mathrm{i} t_{j}}\right)-b\left(e^{\mathrm{i} t_{i}}\right)\right)^{2} \mathrm{~d} t_{1} \mathrm{~d} t_{2} \ldots \mathrm{d} t_{n}
$$


Consequently, if $b$ has the form (8) and $b \in \mathscr{R}$, then

$$
\int_{\pi}^{\pi} \int_{-\pi}^{\pi} \ldots \int_{-\pi}^{\pi} \prod_{1 \leq i<j \leq n}\left(b\left(e^{\mathrm{i} t_{j}}\right)-b\left(e^{\mathrm{i} t_{i}}\right)\right)^{2} \mathrm{~d} t_{1} \mathrm{~d} t_{2} \ldots \mathrm{d} t_{n}>0, \quad \forall n \in \mathbb{N} .
$$

Proof Let $n \in \mathbb{N}$ be fixed. First, by using the definition of the determinant, we get

$$
\operatorname{det} H_{n}=\frac{1}{(2 \pi)^{n}} \int_{\pi}^{\pi} \int_{-\pi}^{\pi} \ldots \int_{-\pi}^{\pi} \operatorname{det} B_{n} \mathrm{~d} t_{1} \mathrm{~d} t_{2} \ldots \mathrm{d} t_{n}
$$

where $B_{n}=D_{n} V_{n}, D_{n}$ is the diagonal matrix with the entries

$$
\left(D_{n}\right)_{j, j}=b^{j-1}\left(e^{\mathrm{i} t_{j}}\right), \quad 1 \leq j \leq n,
$$

and $V_{n}$ is the Vandermonde matrix with the entries

$$
\left(V_{n}\right)_{i, j}=b^{j-1}\left(e^{\mathrm{i} t_{i}}\right), \quad 1 \leq i, j \leq n
$$

By applying the well-known formula for the determinant of the Vandermonde matrix, one arrives at the equation

$$
\begin{aligned}
\operatorname{det} H_{n}= & \frac{1}{(2 \pi)^{n}} \int_{\pi}^{\pi} \int_{-\pi}^{\pi} \ldots \int_{-\pi}^{\pi}\left[\prod_{j=1}^{n} b^{j-1}\left(e^{\mathrm{i} t_{j}}\right)\right] \\
& \times\left[\prod_{1 \leq i<j \leq n}\left(b\left(e^{\mathrm{i} t_{j}}\right)-b\left(e^{\mathrm{i} t_{i}}\right)\right)\right] \mathrm{d} t_{1} \mathrm{~d} t_{2} \ldots \mathrm{d} t_{n} .
\end{aligned}
$$

Second, we apply a symmetrization trick to the identity (23). Let $\sigma$ be a permutation of the set $\{1,2, \ldots, n\}$. Note that the second term in the square brackets in (23), i.e., the Vandermonde determinant, is antisymmetric as a function of variables $t_{1}, t_{2}, \ldots, t_{n}$. Thus, if we change variables in (23) so that $t_{j}=s_{\sigma(j)}$, we obtain

$$
\begin{aligned}
\operatorname{det} H_{n}= & \frac{\operatorname{sign} \sigma}{(2 \pi)^{n}} \int_{\pi}^{\pi} \ldots \int_{-\pi}^{\pi}\left[\prod_{j=1}^{n} b^{j-1}\left(e^{\mathrm{i} s_{\sigma(j)}}\right)\right] \\
& \times\left[\prod_{1 \leq i<j \leq n}\left(b\left(e^{\mathrm{i} s_{j}}\right)-b\left(e^{\mathrm{i} s_{i}}\right)\right)\right] \mathrm{d} s_{1} \ldots \mathrm{d} s_{n} .
\end{aligned}
$$

Now, it suffices to divide both sides of the above equality by $n$ !, sum up over all permutations $\sigma$, and recognize one more Vandermonde determinant on the right-hand side. This yields (21). The second statement of the theorem follows immediately from Corollary 12 and the already proven formula (21). 
Remark 20 Note that

$$
B_{n}\left(z_{1}, \ldots, z_{n}\right):=\prod_{1 \leq i<j \leq n}\left(b\left(z_{j}\right)-b\left(z_{i}\right)\right)^{2} \in \mathbb{C}\left[z_{1}, z_{1}^{-1}, \ldots, z_{n}, z_{n}^{-1}\right],
$$

i.e., $B_{n}\left(z_{1}, \ldots, z_{n}\right)$ is a Laurent polynomial in the indeterminates $z_{1}, \ldots, z_{n}$. The condition (22) tells us that the constant term of $B_{n}\left(z_{1}, \ldots, z_{n}\right)$ has to be positive for all $n \in \mathbb{N}$. Consequently, the inequalities (22) yield a necessary condition for the symbol $b$ of the form (2) to belong to the class $\mathscr{R}$. In principle, these conditions can be formulated as an infinite family of inequalities in terms of the coefficients of $b$ (though in a very complicated form).

We finish this subsection with a discussion about a possible converse of the implication from Corollary 12. It is not clear now, whether, for the symbol $b$ of the form (8), the positive definiteness of all the Hankel matrices $H_{n}$ is a sufficient condition for $b$ to belong to $\mathscr{R}$. If det $H_{n}>0$ for all $n \in \mathbb{N}$, then the Hamburger moment problem with the moment sequence (13) has a solution, say $v$, which is unique. Indeed, the uniqueness follows from the fact that the moment sequence $h_{m}$ does not grow too rapidly as $m \rightarrow \infty$, see [31, Prop. 1.5]. To see this, one has to realize that the spectral radius of $T_{n}(b)$ is majorized by the (spectral) norm of $T_{n}(b)$. This norm can be estimated from above as

$$
\left\|T_{n}(b)\right\| \leq \sum_{k=-r}^{s}\left|a_{k}\right|=: R
$$

Now, taking into account (12), one observes that the moment sequence $h_{m}$ grows at most geometrically since

$$
\left|h_{m}\right| \leq \limsup _{n \rightarrow \infty} \frac{1}{n} \sum_{k=1}^{n}\left|\lambda_{k, n}\right|^{m} \leq R^{m}
$$

where $\lambda_{1, n}, \ldots, \lambda_{n, n}$ stands for the eigenvalues of $T_{n}(b)$ counted repeatedly according to their algebraic multiplicity.

Similarly as in the proof of Lemma 11, one verifies that the $m$-th moment of the limiting measure $\mu$ equals $h_{m}$. Hence, assuming that det $H_{n}>0$ for all $n \in \mathbb{N}$, both measures $\mu$ and $v$ have the same moments. This implies that the Cauchy transforms $C_{\mu}$ and $C_{\nu}$ of measures $\mu$ and $\nu$, respectively, coincide on a neighborhood of $\infty$ since

$$
C_{\mu}(z)=\int_{\mathbb{C}} \frac{\mathrm{d} \mu(x)}{x-z}=-\sum_{m=0}^{\infty} \frac{1}{z^{m+1}} \int_{\mathbb{C}} x^{m} \mathrm{~d} \mu(x)=-\sum_{m=0}^{\infty} \frac{h_{m}}{z^{m+1}}=\int_{\mathbb{R}} \frac{\mathrm{d} v(x)}{x-z}=C_{\nu}(z) .
$$

It can be shown that the equality $C_{\mu}(z)=C_{v}(z)$ hold for all $z \in \mathbb{C},|z|>R$, with $R$ as in (24). This, however, does not imply $\mu=v$.

The measures $\mu$ and $v$ would coincide if $C_{\mu}(z)=C_{v}(z)$ for almost every $z \in \mathbb{C}$ (with respect to the Lebesgue measure in $\mathbb{C}$ ). This can be obtained by imposing an 


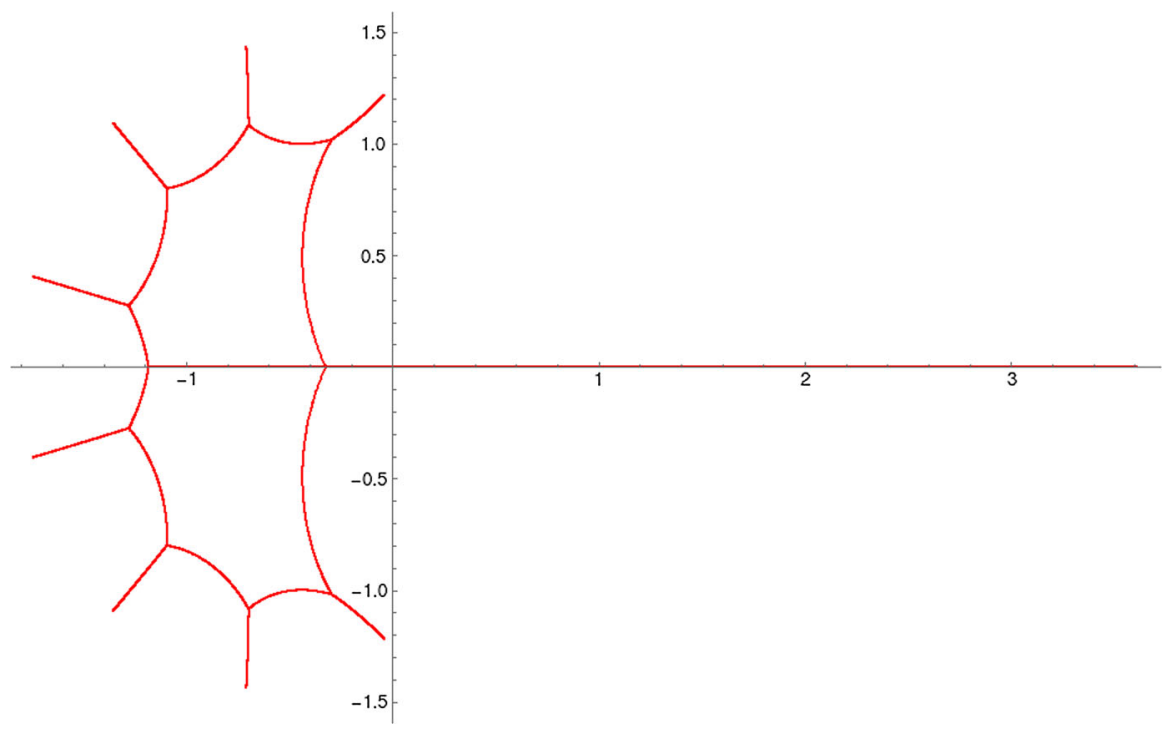

Fig. 2 The set $\Lambda(b)$ for $b(z)=1 / z+\sum_{k=1}^{10} k z^{k}$, which seems to separate the plane

additional assumption on $\Lambda(b)$ requiring its complement to be connected. Indeed, since the Cauchy transform of a measure is analytic outside the support of the measure, the equality $C_{\mu}(z)=C_{v}(z)$ can be extended to all $z \notin \Lambda(b) \cup$ supp $v$ by analyticity provided that $\mathbb{C} \backslash \Lambda(b)$ is connected. Clearly, both sets $\Lambda(b)$ and supp $v$ have zero Lebesgue measure and therefore $\mu=v$.

It follows from the above discussion that, if there exists $b$ of the form (2) such that det $H_{n}>0$ for all $n \in \mathbb{N}$, and $b \notin \mathscr{R}$, then the set $\mathbb{C} \backslash \Lambda(b)$ has to be disconnected. Let us stress that it is by no means clear for which symbols $b$ the set $\mathbb{C} \backslash \Lambda(b)$ is connected. To our best knowledge, the only settled case corresponds to symbols $b$ that are trinomials when $\mathbb{C} \backslash \Lambda(b)$ is known to be connected as pointed out in [27]. On the other hand, the relatively simple examples of $b$ where $\Lambda(b)$ separates the plane $\mathbb{C}$ are known, see [4, Prop. 5.2]. One might believe that, if $T_{n}(b)$ is a lower (or upper) Hessenberg matrix, i.e., $r=1$ (or $s=1$ ) in (8), then $\mathbb{C} \backslash \Lambda(b)$ is connected. It seems that it is not the case either. It is not our intention to prove it analytically; we provide only a numerical evidence given by Fig. 2 .

\subsection{Associated Jacobi Operator and Orthogonal Polynomials}

The aim of this subsection is to summarize several properties of the Jacobi operator whose spectral measure is given by the limiting measure for $b \in \mathscr{R}$ in the form (8). Here we denote by $\ell^{2}(\mathbb{N})$ the Hilbert space of square summable sequences indexed by $\mathbb{N}$, by $\langle\cdot, \cdot\rangle$ the standard inner product on $\ell^{2}(\mathbb{N})$, and by $\left\{e_{k} \mid k \in \mathbb{N}\right\}$ the canonical basis of $\ell^{2}(\mathbb{N})$.

Suppose that $b \in \mathscr{R}$ is of the form (8). By Lemma 11, the limiting measure $\mu$ is the unique solution to a determinate Hamburger moment problem. Consequently, there is 
a unique self-adjoint Jacobi operator $J(b)$ acting on $\ell^{2}(\mathbb{N})$ whose spectral measure $E_{J(b)}$ is related to the limiting measure $\mu$ by the formula

$$
\mu=\left\langle e_{1}, E_{J(b)} e_{1}\right\rangle .
$$

Moreover, the spectrum of $J(b)$ coincides with the support of $\mu$ that equals $\Lambda(b)$. These results follow from the well-known theory and can be found, for example, in [2].

In the spectral theory of Jacobi operators, the crucial role is played by the Weyl $\mathrm{m}$ function defined as the first diagonal element of the resolvent operator; see [32, Chp.2]. In our case, we write $m_{b}(\lambda):=\left\langle e_{1},(J(b)-\lambda)^{-1} e_{1}\right\rangle$ for $\lambda \notin \Lambda(b)$. By expressing the resolvent operator of $J(b)$ in terms of its spectral measure and using (25), one obtains

$$
m_{b}(\lambda)=\int_{\Lambda(b)} \frac{\mathrm{d} \mu(x)}{x-\lambda}, \quad \lambda \notin \Lambda(b) .
$$

In other words, the Weyl $m$-function of $J(b)$ is nothing but the Cauchy transform of the limiting measure $\mu$. Now, one can make use of the expression (16) getting the noteworthy formula

$$
m_{b}(\lambda)=\frac{1}{\pi} \int_{0}^{\pi} \frac{\mathrm{d} t}{b(\gamma(t))-\lambda}
$$

for all $\lambda \notin \Lambda(b)$, provided that $\gamma$ is parametrized as in (9). The density of $\mu$ can be recovered from $m_{b}$ with the aid of the Stieltjes-Perron inversion formula

$$
\frac{\mathrm{d} \mu}{\mathrm{d} x}(x)=\frac{1}{\pi} \lim _{\epsilon \rightarrow 0+} \operatorname{Im} m_{b}(x+\mathrm{i} \epsilon)
$$

for all $x \in \Lambda(b)$ that are not exceptional points; see, for example, [32, Chp. 2 and Append. B].

Further, we turn our attention to the inverse spectral problem which, in general, asks for a reconstruction of an operator if certain spectral quantities are known. In the case of Jacobi operator $J(b)$, the given quantity is the measure $\mu$ determined by $b \in \mathscr{R}$ and related to $J(b)$ via (25). The reconstruction of $J(b)$ can be made by means of the diagonal sequence $b_{n}:=\left\langle e_{n}, J(b) e_{n}\right\rangle, n \in \mathbb{N}$, and the off-diagonal sequence $a_{n}:=\left\langle e_{n+1}, J(b) e_{n}\right\rangle, n \in \mathbb{N}$, that appears in the tridiagonal matrix representation of $J(b)$. This initiates a study of the mapping that sends $b \in \mathscr{R}$ of the form (8) to the corresponding Jacobi parameters $\left\{a_{n}\right\}_{n=1}^{\infty}$ and $\left\{b_{n}\right\}_{n=1}^{\infty}$.

In general, the Jacobi parameters are expressible in terms of the moment sequence $\left\{h_{m}\right\}_{m=0}^{\infty}$ by the well-known formulas [32, Eq. (2.118)]

$$
a_{1}=\frac{\sqrt{\operatorname{det} H_{2}}}{\operatorname{det} H_{1}}=\sqrt{h_{2}-h_{1}^{2}}, \quad a_{n}=\frac{\sqrt{\operatorname{det} H_{n-1} \operatorname{det} H_{n+1}}}{\operatorname{det} H_{n}}, \quad n \geq 2,
$$


and

$$
b_{1}=\frac{\operatorname{det} \tilde{H}_{1}}{\operatorname{det} H_{1}}=h_{1}, \quad b_{n}=\frac{\operatorname{det} \tilde{H}_{n}}{\operatorname{det} H_{n}}-\frac{\operatorname{det} \tilde{H}_{n-1}}{\operatorname{det} H_{n-1}}, \quad n \geq 2,
$$

where $H_{n}$ is the Hankel matrix (11) and $\tilde{H}_{n}$ is obtained from $H_{n+1}$ by deleting its $n$th row and its $(n+1)$ st column. However, it is unlikely that in a concrete situation, one can use general formulas (27) and (28) to obtain the Jacobi parameters explicitly. In Sect. 4, this is done only in two examples related to a tridiagonal and 4-diagonal Toeplitz matrix. Therefore we provide the following statement that gives the leading term of the asymptotic expansion of $a_{n}$ and $b_{n}$, as $n \rightarrow \infty$.

Theorem 21 Let $b \in \mathscr{R}$ of the form (8) and $\Lambda(b)=[\alpha, \beta]$. Then $J(b)$ is a compact perturbation of the Jacobi matrix with the constant diagonal sequence $(\alpha+\beta) / 2$ and the constant off-diagonal sequence $(\beta-\alpha) / 4$, i.e.,

$$
\lim _{n \rightarrow \infty} a_{n}=\frac{\beta-\alpha}{4} \text { and } \lim _{n \rightarrow \infty} b_{n}=\frac{\alpha+\beta}{2} .
$$

Remark 22 Let us recall that Theorems 13 and 16 show how to determine the endpoints $\alpha$ and $\beta$ of $\Lambda(b)$ with the aid of the Jordan curve $\gamma$ from $b^{-1}(\mathbb{R})$.

Proof of Theorem 21 The statement is a consequence of Rakhmanov's theorem [12, Thm. 4]; see also Thm. 1 in loc. cit. referring to a weaker older result due to P. Nevai [25] that is still sufficient for our purposes. The latter theorem implies that if

(i) $J$ is a bounded self-adjoint Jacobi operator such that $\operatorname{spec}_{\mathrm{ess}}(J)=[a, b]$,

(ii) $\rho(x)>0$ for almost every $x \in[a, b]$, where $\rho$ denotes the density of the Lebesgue absolutely continuous component of the measure $\left\langle e_{1}, E_{J} e_{1}\right\rangle, E_{J}$ being the spectral measure of $J$,

then $J$ is a compact perturbation of the Jacobi operator with constant diagonal sequence $(a+b) / 2$ and constant off-diagonal sequence $(b-a) / 4$.

In case of $J(b)$, we have $\operatorname{spec}(J(b))=\Lambda(b)=[\alpha, \beta]$. In fact, since $\operatorname{spec}(J(b))$ does not contain isolated points, the discrete part of the spectrum of the self-adjoint operator $J(b)$ is empty and hence $\operatorname{spec}_{\text {ess }}(J(b))=[\alpha, \beta]$. Taking into account (25), it suffices to show that the density of the limiting measure $\mu$ is positive almost everywhere on $(\alpha, \beta)$. The latter fact is true since the density is positive on every analytic arc of $\Lambda(b)$, i.e., everywhere except possibly at the exceptional points which are finitely many, see [19, Cor. 4c].

Remark 23 Some aspects discussed for the Jacobi operator $J(b)$ above can be reformulated for the corresponding family of orthogonal polynomials as follows. For any $b \in \mathscr{R}$ of the form (8), there exists a family of orthogonal polynomials $\left\{p_{n}\right\}_{n=0}^{\infty}$, determined by the three-term recurrence

$$
p_{n+1}(x)=\left(x-b_{n+1}\right) p_{n}(x)-a_{n}^{2} p_{n-1}(x), \quad n \in \mathbb{N}_{0},
$$


with the initial conditions $p_{-1}(x)=0$ and $p_{0}(x)=1$, where the coefficients $\left\{a_{n}\right\}_{n=1}^{\infty}$ and $\left\{b_{n}\right\}_{n=1}^{\infty}$ are given by (27) and (28) ( $a_{0}$ is arbitrary). This family satisfies the orthogonality relation

$$
\int_{\alpha}^{\beta} p_{n}(x) p_{m}(x) \rho(x) \mathrm{d} x=\frac{\operatorname{det} H_{n+1}}{\operatorname{det} H_{n}} \delta_{m, n}, \quad m, n \in \mathbb{N}_{0},
$$

where $\rho$ stands for the density of the limiting measure $\mu$ supported on $\Lambda(b)=[\alpha, \beta]$. For $n=0$, one has to set det $H_{0}:=1$ in (30). In the terminology of orthogonal polynomials, the statement of Theorem 21 says that the family of orthogonal polynomials $\left\{p_{n}\right\}_{n=0}^{\infty}$ belongs to the Blumenthal-Nevai class $M((\beta-\alpha) / 2,(\alpha+\beta) / 2)$, see [25]. Particular examples of these families of polynomials are examined in Sect. 4.

\section{Examples and Numerical Computations}

\subsection{Example 1 (Tridiagonal Case)}

First, we take a look at the simplest nontrivial situation when $T(b)$ is a tridiagonal Toeplitz matrix. Since the entry on the main diagonal only causes a shift of the spectral parameter and the matrix $T(b)$ itself can be scaled by a nonzero constant, we may assume, without loss of generality, that $T(b)$ belongs to the one-parameter family of tridiagonal Toeplitz matrices with the symbol

$$
b(z)=\frac{1}{z}+a z
$$

where $a \in \mathbb{C} \backslash\{0\}$.

First, we decide for what parameter $a$, the symbol $b \in \mathscr{R}$. By Theorem 1 , $\operatorname{spec} T_{2}(b) \subset \mathbb{R}$ is a necessary condition from which one easily deduces that $a>0$, if $b \in \mathscr{R}$. Next, for $a>0$, the symbol $b$ is real-valued on the circle $\gamma(t)=a^{-1 / 2} e^{\mathrm{i} t}$, $t \in[-\pi, \pi]$. Thus, by using Theorem 1 once more, we conclude that $b \in \mathscr{R}$ if and only if $a>0$.

The limiting density is derived in [19, Sec.5] and reads

$$
\frac{\mathrm{d} \mu}{\mathrm{d} x}(x):=\frac{1}{\pi \sqrt{4 a-x^{2}}}, \quad x \in(-2 \sqrt{a}, 2 \sqrt{a}),
$$

for $a>0$. Alternatively, the formula (31) can be readily deduced by using Theorem 16 .

Further, let us examine the structure of the Jacobi matrix $J(b)$ and the corresponding family of orthogonal polynomials. First, since the density (31) is an even function on the interval symmetric with respect to 0 , the diagonal sequence $\left\{b_{n}\right\}_{n=1}^{\infty}$ vanishes, as it follows, for example, from [7, Thm. 4.2 (c)]. To compute the off-diagonal sequence $\left\{a_{n}\right\}_{n=1}^{\infty}$, we need to evaluate det $H_{n}$, where $H_{n}$ is the Hankel matrix (11) with the entries given by the moments 


$$
h_{m}=\frac{1}{2 \pi} \int_{-\pi}^{\pi}\left(e^{-\mathrm{i} t}+a e^{\mathrm{i} t}\right)^{m} \mathrm{~d} t= \begin{cases}\left(\begin{array}{c}
2 k \\
k
\end{array}\right) a^{k} & \text { if } m=2 k, \\
0 & \text { if } m=2 k-1 .\end{cases}
$$

The evaluation of det $H_{n}$ is treated in Lemma 27 in the Appendix. By using Lemma 27 together with (27), one immediately gets $a_{1}=2 \sqrt{a}$ and $a_{n}=\sqrt{a}$ for $n>1$. Thus, the self-adjoint Jacobi operator associated with $b$ has the matrix representation

$$
J(b)=\left(\begin{array}{cccccc}
0 & 2 \sqrt{a} & & & & \\
2 \sqrt{a} & 0 & \sqrt{a} & & & \\
& \sqrt{a} & 0 & \sqrt{a} & & \\
& & \sqrt{a} & 0 & \sqrt{a} & \\
& & & \ddots & \ddots & \ddots
\end{array}\right),
$$

where $a>0$.

The corresponding family of orthogonal polynomials $\left\{p_{n}\right\}_{n=0}^{\infty}$ generated by the recurrence (29) (and initial conditions given therein) satisfies the orthogonality relation

$$
\int_{-2 \sqrt{a}}^{2 \sqrt{a}} p_{n}(x) p_{m}(x) \frac{\mathrm{d} x}{\sqrt{4 a-x^{2}}}=\pi\left(2-\delta_{n, 0}\right) a^{n} \delta_{n, m}, \quad \forall m, n \in \mathbb{N}_{0}
$$

which one verifies by using (30) together with the formula (31) and Lemma 27. Polynomials $\left\{p_{n}\right\}_{n=0}^{\infty}$ do not belong to any family listed in the hypergeometric Askey scheme [21]. However, they can be written as the following linear combination of Chebyshev polynomials of the second kind,

$$
p_{n}(x)=a^{n / 2}\left(U_{n}\left(\frac{x}{2 \sqrt{a}}\right)-3 U_{n-2}\left(\frac{x}{2 \sqrt{a}}\right)\right)
$$

for $n \in \mathbb{N}$ (here $U_{-1}(x):=0$ ). The above equation and the hypergeometric representation of Chebyshev polynomials, see [21, Eq. (9.8.36)], can be used to obtain the explicit formula

$$
p_{n}(x)=\sum_{k=0}^{\left\lfloor\frac{n}{2}\right\rfloor}(-1)^{k} \frac{(n+2 k)(n-1-k) !}{k !(n-2 k) !} a^{k} x^{n-2 k}, \quad n \in \mathbb{N},
$$

where $\lfloor y\rfloor$ denotes the greatest integer less than or equal to a real number $y$.

\subsection{Example 2 (4-Diagonal Case)}

Let us examine the case of symbols (2) with $r=1$ and $s=2$. Without loss of generality, we can set $a_{-1}=1$ and $a_{0}=0$, which yields the symbol $b$ of the form 


$$
b(z)=\frac{1}{z}+\alpha z+\beta z^{2},
$$

where $\alpha \in \mathbb{C}$ and $\beta \in \mathbb{C} \backslash\{0\}$.

First, we discuss for which parameters $\alpha$ and $\beta$ we have $b \in \mathscr{R}$. By Remark 10, if $b \in \mathscr{R}, \alpha$ and $\beta$ have to be real. Further, according to Theorem 1 , if $b \in \mathscr{R}$, then $\operatorname{spec} T_{3}(b) \subset \mathbb{R}$. The characteristic polynomial of $T_{3}(b)$ reads

$$
\operatorname{det}\left(T_{3}(b)-z\right)=\beta+2 \alpha z-z^{3} .
$$

By inspection of the discriminant of the above cubic polynomial with real coefficients, one concludes that its roots are real if and only if $\alpha^{3} \geq 27 \beta^{2}$.

Next, we show that, for $\beta \in \mathbb{R} \backslash\{0\}$ and $\alpha^{3} \geq 27 \beta^{2}, b^{-1}(\mathbb{R})$ contains a Jordan curve. Note that, if $\alpha^{3} \geq 27 \beta^{2}$, the equation

$$
z^{2} b^{\prime}(z)=-1+\alpha z^{2}+2 \beta z^{3}=0
$$

has all roots nonzero real and they cannot degenerate to a triple root. Consequently, all critical points of $b$ are real. At the same time, these points are the intersection points of the net $n_{b}$. Taking into account that only one curve in $n_{b}$ (the real line) passes through 0 , because $b(z) \sim 1 / z$ as $z \rightarrow 0$, and two curves (the extended real line and one more) pass through $\infty$, because $b(z) \sim \beta z^{2}$ as $z \rightarrow \infty$, one concludes that there has to be another arc in $b^{-1}(\mathbb{R})$ passing through a real critical point of $b$. This arc necessarily closes into a Jordan curve located in $b^{-1}(\mathbb{R})$. Altogether, Theorem 1 implies that $b \in \mathscr{R}$ if and only if $\beta \in \mathbb{R} \backslash\{0\}$ and $\alpha^{3} \geq 27 \beta^{2}$.

Next, we will derive the limiting measure $\mu$ explicitly in a special case when the symbol takes the form

$$
b(z)=\frac{1}{z}(1+a z)^{3},
$$

where $a \in \mathbb{R} \backslash\{0\}$. This corresponds to $\alpha=3 a^{2}, \beta=a^{3}$ in (33), but we additionally add a real constant to $b$. Clearly, if we add a real constant to $b$, the net $n_{b}$ does not change and $\Lambda(b)$ is just shifted by the constant. Hence, we may use the previous discussion to conclude that $b$, given by (34), belongs to the class $\mathscr{R}$ for all $a \in \mathbb{R} \backslash\{0\}$.

Note that the critical points of $b$ are $-1 / a$ and $1 /(2 a)$; hence the measure $\mu$ is supported on the closed interval with the endpoints $b(-1 / a)=0$ and $b(1 /(2 a))=$ $27 a / 4$. By using the binomial formula, one verifies that the constant term of $b^{m}(z)$ equals

$$
h_{m}=\left(\begin{array}{c}
3 m \\
m
\end{array}\right) a^{m}, \quad m \in \mathbb{N}_{0} .
$$

The generating function for the moments $h_{m}$ can be expressed in terms of the Gauss hypergeometric series as 


$$
\sum_{m=0}^{\infty} h_{m} z^{m}={ }_{2} F_{1}\left(\frac{1}{3}, \frac{2}{3} ; \frac{1}{2} ; \frac{27}{4} a z\right)
$$

For the sake of simplicity of the forthcoming formulas and without loss of generality, we set $a=4 / 27$. We can apply the identity

$$
{ }_{2} F_{1}\left(c, 1-c ; \frac{1}{2} ;-z\right)=\frac{(\sqrt{1+z}+\sqrt{z})^{2 c-1}+(\sqrt{1+z}-\sqrt{z})^{2 c-1}}{2 \sqrt{1+z}},
$$

valid for $c \in(0,1)$ and $|z|<1$, see [26, Eq.7.3.3.4, p. 486], to the right-hand side of (35) with $c=2 / 3$ and deduce the formula for the Weyl $m$-function of the Jacobi operator $J(b)$ which reads

$m_{b}(z)=-\frac{1}{z}{ }_{2} F_{1}\left(\frac{1}{3}, \frac{2}{3} ; \frac{1}{2} ; \frac{1}{z}\right)=-\frac{\left(\frac{\mathrm{i}}{\sqrt{z}}+\sqrt{1-\frac{1}{z}}\right)^{1 / 3}+\left(-\frac{\mathrm{i}}{\sqrt{z}}+\sqrt{1-\frac{1}{z}}\right)^{1 / 3}}{2 \sqrt{z^{2}-z}}$

for $z \in \mathbb{C} \backslash[0,1]$. By evaluating the limit in (26), one obtains

$$
\frac{\mathrm{d} \mu}{\mathrm{d} x}(x)=\frac{\sqrt{3}}{4 \pi} \frac{(1+\sqrt{1-x})^{1 / 3}+(1-\sqrt{1-x})^{1 / 3}}{x^{2 / 3} \sqrt{1-x}}, \quad x \in(0,1) .
$$

This density appeared earlier in connection with Faber polynomials [22]; see also $[8,13]$.

Next, we examine the operator $J(b)$ and the corresponding family of orthogonal polynomials in detail. First, we derive formulas for the diagonal sequence $\left\{b_{n}\right\}_{n=1}^{\infty}$ and off-diagonal sequence $\left\{a_{n}\right\}_{n=1}^{\infty}$ of $J(b)$. In [14], the Hankel determinant

$$
\operatorname{det} H_{n}=3^{n-1}\left(\prod_{i=0}^{n-1} \frac{(3 i+1)(6 i) !(2 i) !}{(4 i) !(4 i+1) !}\right) a^{n(n-1)}
$$

has been evaluated with $a=1$, see [14, Eq. (1)]. The slightly more general identity (38) with the additional parameter $a$ can be justified by using the same argument as in the first paragraph of the proof of Lemma 27. Moreover, the first equation from [14, Eq. (25)] yields

$$
\operatorname{det} \tilde{H}_{n}=\frac{27 n^{2}-8 n-1}{2(4 n-1)} \operatorname{det} H_{n}, \quad n \in \mathbb{N},
$$

(in the notation used in [14, Eq. (25)], $H_{1} \equiv H_{1}(n, 0)$ coincides with det $\tilde{H}_{n+1}$ and $H_{0} \equiv H_{0}(n, 0)$ coincides with $\left.\operatorname{det} H_{n+1}\right)$. 
By substituting the identities (38) and (39) in the general formulas (27) and (28), one obtains

$$
a_{1}^{2}=6 a^{2} \text { and } a_{k}^{2}=\frac{9(6 k-5)(6 k-1)(3 k-1)(3 k+1)}{4(4 k-3)(4 k-1)^{2}(4 k+1)} a^{2} \text { for } k>1,
$$

and

$$
b_{1}=3 a \text { and } b_{k}=\frac{3\left(36 k^{2}-54 k+13\right)}{2(4 k-5)(4 k-1)} a \text { for } k>1 \text {. }
$$

The corresponding family of monic orthogonal polynomials generated by the recurrence (29) is orthogonal with respect to the density (37) for $a=4 / 27$. Taking into account (30) and the identity (38), one gets the orthogonality relation

$$
\int_{0}^{1} p_{n}(x) p_{m}(x) \rho(x) \mathrm{d} x=\left(3-2 \delta_{n, 0}\right)\left(\frac{4}{27}\right)^{2 n} \frac{(3 n+1)(6 n) !(2 n) !}{(4 n) !(4 n+1) !} \delta_{n, m}, \quad \forall m, n \in \mathbb{N}_{0}
$$

The polynomial sequence $\left\{p_{n}\right\}_{n=0}^{\infty}$ does not coincide with any family listed in the Askey scheme [21] either. Nevertheless, $p_{n}$ can be expressed as a linear combination of the associated Jacobi polynomials introduced and studied by J. Wimp in [36]. Following notation from [36], the associated Jacobi polynomials $P_{n}^{(\alpha, \beta)}(x ; c)$ constitute a three-parameter family of orthogonal polynomials generated by the same recurrence as the Jacobi polynomials $P_{n}^{(\alpha, \beta)}(x)$, but every occurrence of $n$ in the coefficients of the recurrence relation defining the Jacobi polynomials is replaced by $n+c$, see [36, Eq. (12)]. Set

$$
r_{n}^{(\alpha, \beta)}(x ; c):=\frac{2^{n}(c+\alpha+\beta+1)_{n}(c+1)_{n}}{(2 c+\alpha+\beta+1)_{2 n}} P_{n}^{(\alpha, \beta)}(2 x-1 ; c), \quad n \in \mathbb{N}_{0},
$$

and $r_{-1}^{(\alpha, \beta)}(x ; c):=0$, where $(x)_{n}=x(x+1) \ldots(x+n-1)$ is the Pochhammer symbol. Then, putting again $a=4 / 27$, one has

$$
2^{n} p_{n}(x)=r_{n}^{(\alpha, \beta)}(x ; c)-\frac{4}{27} r_{n-1}^{(\alpha, \beta)}(x ; c+1)-\frac{256}{729} r_{n-2}^{(\alpha, \beta)}(x ; c+2), \quad n \in \mathbb{N},
$$

where $\alpha=1 / 2, \beta=-2 / 3$, and $c=-1 / 6$. Verification of (40) is done in a completely routine way by showing that both sides satisfy the same recurrence relation with the same initial conditions.

Finally, since Wimp derived the explicit formula for associated Jacobi polynomials in [36, Theorem 1], one can make use of this result together with (40) to compute the explicit expression for $p_{n}(x)$. However, the computation is somewhat lengthy and the resulting formula is rather cumbersome; we omit the details and state only the final 
result for the record. For $n \in \mathbb{N}_{0}$, one has

$$
p_{n}(x)=A_{n} \sum_{k=0}^{n} B_{n}(k) C_{n}(k) x^{k}
$$

where

$$
A_{n}=(-1)^{n} \frac{\left(\frac{1}{6}\right)_{n}\left(\frac{5}{6}\right)_{n}}{5\left(n+\frac{1}{2}\right)_{n} n !}, \quad B_{n}(k)=\frac{(-n)_{k}\left(n+\frac{1}{2}\right)_{k}}{\left(\frac{1}{6}\right)_{k}\left(\frac{5}{6}\right)_{k}}
$$

and

$$
C_{n}(k)=\sum_{i=0}^{n-k} \frac{(k-n)_{i}\left(n+k+\frac{1}{2}\right)_{i}\left(-\frac{5}{6}\right)_{i}\left(-\frac{1}{6}\right)_{i}}{\left(-\frac{1}{2}\right)_{i}\left(k+\frac{1}{6}\right)_{i}\left(k+\frac{5}{6}\right)_{i}} \frac{(6 i-5)(18 i+1)}{(2 i-1)(2 i+1)}
$$

\subsection{Example 3}

Both cases treated in the previous subsections, where the limiting measure was derived fully explicitly, can be thought of as special cases of the more general symbol

$$
b(z)=\frac{1}{z^{r}}(1+a z)^{r+s}
$$

(up to a shift by a constant term), where $r, s, \in \mathbb{N}$ and $a \in \mathbb{R} \backslash\{0\}$. By using the binomial formula, one computes

$$
h_{m}=\frac{1}{2 \pi} \int_{0}^{2 \pi} b^{m}\left(e^{\mathrm{i} \theta}\right) \mathrm{d} \theta=\left(\begin{array}{c}
(r+s) m \\
r m
\end{array}\right) a^{r m}, \quad m \in \mathbb{N}_{0} .
$$

First, we prove that $b \in \mathscr{R}$ for all $r, s \in \mathbb{N}$ and $a \in \mathbb{R} \backslash\{0\}$ by showing that $b^{-1}(\mathbb{R})$ contains a Jordan curve. Without loss of generality, we may again put $a=1$ (otherwise one can take the $1 / a$ multiple of the curve $\gamma$ given below). Define

$$
\gamma(t):=\frac{\sin \omega t}{\sin (1-\omega) t} e^{\mathrm{i} t}, \quad t \in(-\pi, \pi]
$$

where $\omega:=r /(r+s)$. The value $\gamma(0)=r / s$ is determined by the corresponding limit. Figure 3 shows the Jordan $\gamma$ for three special choices of $r$ and $s$. Expressing the sine function in terms of complex exponentials, one easily verifies that

$$
b(\gamma(t))=\frac{\sin ^{r+s} t}{\sin ^{r}(\omega t) \sin ^{s}((1-\omega) t)}, \quad t \in(-\pi, \pi],
$$




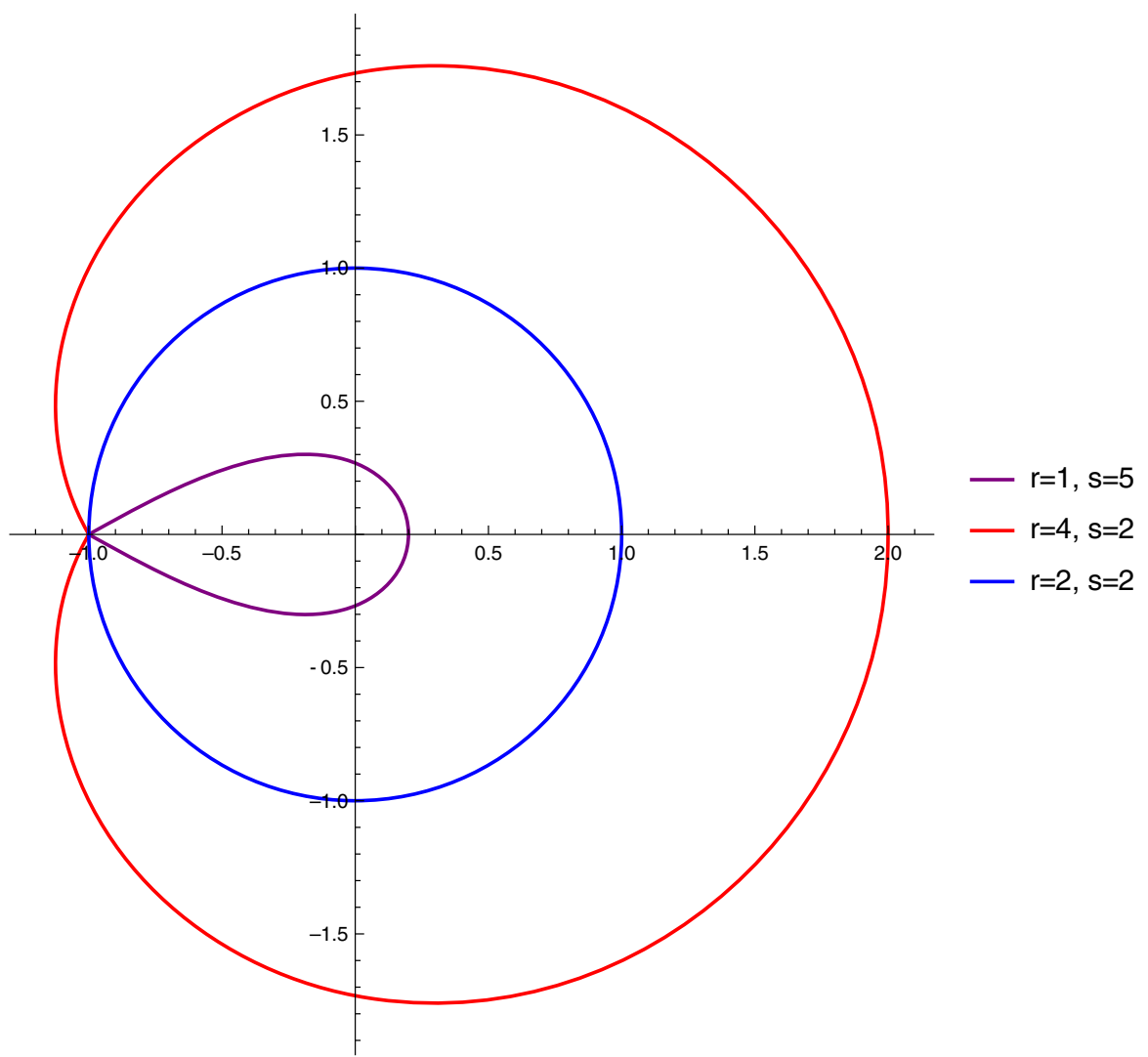

Fig. 3 Illustration for Example 3: The plots of the Jordan curves $\gamma$ given by (42) for 3 particular choices of parameters $r$ and $s$ (Color figure online)

where the value at $t=0$ is the respective limit. Thus, $b$ restricted to the image of $\gamma$ is real-valued and Theorem 1 implies $b \in \mathscr{R}$.

The parametrization (42) has the polar form (9). Moreover, $b$ has no critical point on the arc of $\gamma$ in the upper half-plane since

$$
b^{\prime}(\gamma(t))=\frac{s \gamma(t)-r}{(1+\gamma(t)) \gamma(t)} b(\gamma(t)) \neq 0, \quad \forall t \in(0, \pi)
$$

as one readily verifies. Taking into account that

$$
b(\gamma(0))=\frac{(r+s)^{r+s}}{r^{r} s^{s}} \quad \text { and } \quad b(\gamma(\pi))=0,
$$

we see that $b \circ \gamma$ is strictly decreasing on $(0, \pi)$. Consequently, by Theorem 16 , the limiting mesure is supported on the interval

$$
\operatorname{supp} \mu=\left[0, r^{-r} s^{-s}(r+s)^{r+s}\right] \text {. }
$$



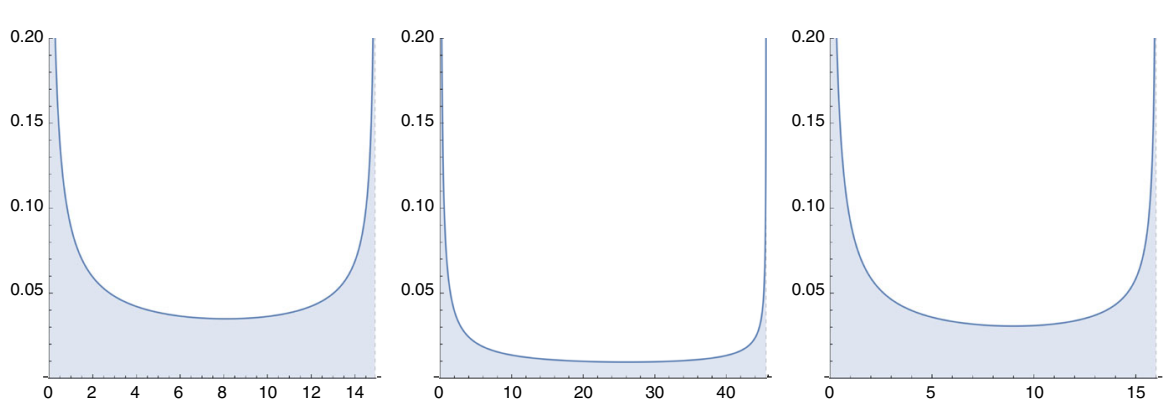

Fig. 4 Illustration for Example 3: The plots of the density of $\mu$ for $r=1, s=5$ (left), $r=4, s=2$ (center), and $r=s=2$ (right)

and, according to (18), the distribution function of $\mu$ satisfies

$$
F_{\mu}(b(\gamma(t)))=1-\frac{t}{\pi} \quad \text { for } 0 \leq t \leq \pi
$$

It seems that, for general parameters $r$ and $s$, the density of $\mu$ cannot be expressed explicitly because the function in (43) cannot be inverted; see Fig. 4 for numerical plots of the density of $\mu$. Let us point out that, besides the cases $r=1$ and either $s=1$ or $s=2$, one can also derive explicitly the density for the self-adjoint 5-diagonal case, i.e., $r=s=2(a=1)$. The resulting formula reads

$$
\rho(x)=\frac{\sqrt{4+\sqrt{x}}}{2 \pi x^{3 / 4} \sqrt{16-x}}, \quad x \in(0,16),
$$

since the Weyl $m$-function can be expressed as

$$
m_{b}(z)=-\frac{1}{z} \sqrt{\frac{1+\sqrt{1-16 / z}}{2(1-16 / z)}}, \quad z \in \mathbb{C} \backslash[0,16] .
$$

The above formula follows from the generating function of $\left\{h_{m}\right\}_{m=0}^{\infty}$ which, for $|z|<$ $1 / 16$, reads

$$
\begin{aligned}
\sum_{m=0}^{\infty}\left(\begin{array}{l}
4 m \\
2 m
\end{array}\right) z^{m} & ={ }_{2} F_{1}\left(\frac{1}{4}, \frac{3}{4} ; \frac{1}{2} ; 16 z\right)=\frac{\sqrt{\sqrt{1-16 z}+4 i \sqrt{z}+\sqrt{\sqrt{1-16 z}-4 i \sqrt{z}}}}{2 \sqrt{1-16 z}} \\
& =\sqrt{\frac{1+\sqrt{1-16 z}}{2(1-16 z)}}
\end{aligned}
$$

where we make use of the identity (36) with $c=3 / 4$.

Derivation of a closed formula for the sequences $\left\{a_{n}\right\}_{n=1}^{\infty}$ and $\left\{b_{n}\right\}_{n=1}^{\infty}$ determining the Jacobi operator $J(b)$ for general values of $r$ and $s$ seems to be out of our reach. 
Even a closed expression for the determinant of the Hankel matrix $H_{n}$ determined by the sequences

$$
h_{m}=\left(\begin{array}{c}
4 m \\
m
\end{array}\right) \quad \text { or } \quad h_{m}=\left(\begin{array}{c}
4 m \\
2 m
\end{array}\right)
$$

corresponding to the special cases with $a=1, r=1$, and $s=3$ or $r=2$ and $s=2$, respectively, is unknown, to the best of our knowledge. In fact, numerical experiments with the sequence of these determinants show a presence of huge prime factors which might indicate that no closed formula, similar to (38), exists for the Hankel determinants. Nevertheless, Theorem 21 and equation (44) yield the limit formulas

$$
2 \lim _{n \rightarrow \infty} a_{n}=\lim _{n \rightarrow \infty} b_{n}=\frac{(r+s)^{r+s}}{2 r^{r} s^{s}}
$$

for all $r, s \in \mathbb{N}$.

\subsection{More General Examples Based on Example 3}

Most of the results of this paper are devoted to banded Toeplitz matrices but Theorem 8 is applicable to matrices with more general symbols. A combination of the previous example with the symbol (41) and Theorem 8 provides us with more examples of possibly non-self-adjoint Toeplitz matrices given by a more general symbol. For instance, one can proceed as follows.

If $f$ is a function analytic on $\mathbb{C} \backslash\{0\}$ which maps $\mathbb{R} \backslash\{0\}$ to $\mathbb{R}$, and $b$ is as in (41), then the symbol $a:=f \circ b$ satisfies the assumptions of Theorem 8 with the Jordan curve given by (42). Consequently, $\operatorname{spec} T_{n}(a) \subset \mathbb{R}$ for all $n \in \mathbb{N}$. To be even more concrete, the possible choices of $f$ comprise, e.g., $f \in\{\exp , \sin , \cos , \sinh , \cosh , \ldots\}$, producing many examples of non-self-adjoint and non-banded Toeplitz matrices whose principal submatrices have purely real spectrum.

\subsection{Various Numerical Experiments}

To illustrate a computational applicability of Theorems 13 and 16, we add two more complicated examples treated numerically using Wolfram Mathematica. In particular, to emphasize the connection between the existence of a Jordan curve in $b^{-1}(\mathbb{R})$ and the reality of the limiting set of eigenvalues of the corresponding Toeplitz matrices, we provide some numerical plots on this account.

\subsubsection{Example 4}

We plot the numerically obtained density by applying Theorem 16 to the symbol

$$
b(z)=\frac{1}{z^{3}}-\frac{1}{z^{2}}+\frac{7}{z}+9 z-2 z^{2}+2 z^{3}-z^{4},
$$

whose net is shown in Fig. 1 (left). 


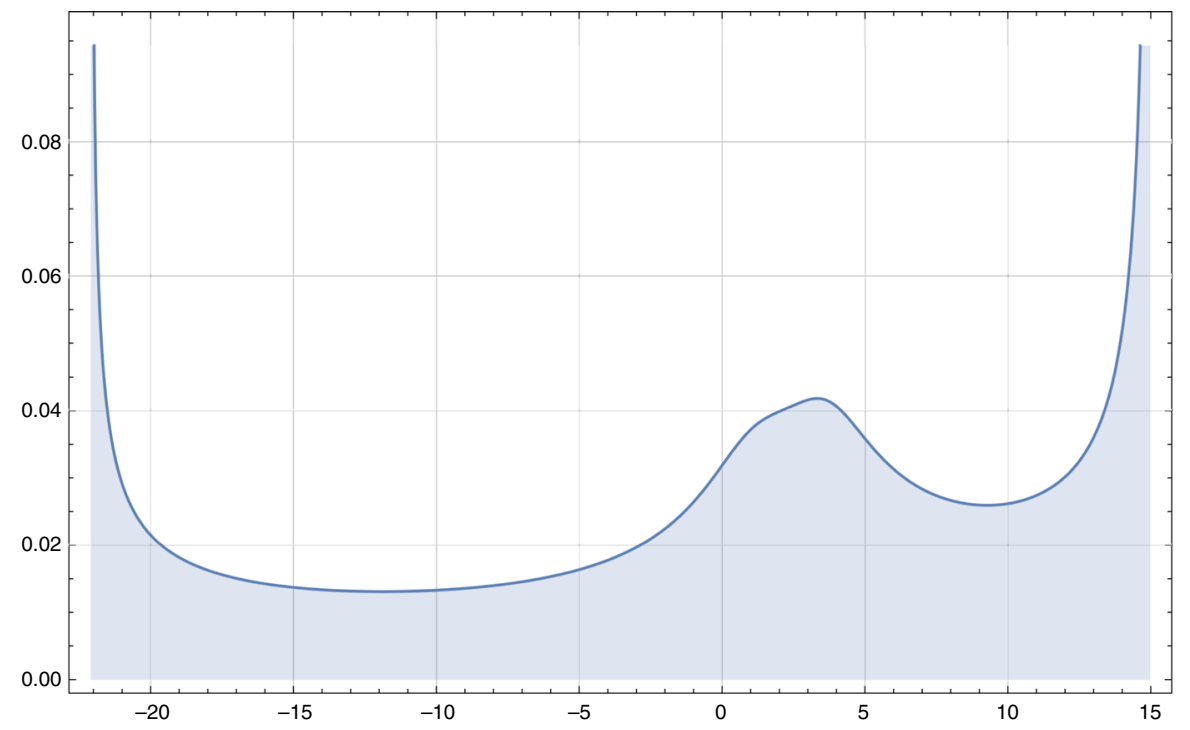

Fig. 5 Illustration for Example 4: The plot of the density of $\mu$

Although we can only check the validity of the assumptions of Theorem 16 numerically, looking at Fig. 1 it is reasonable to believe the Jordan curve contained in $b^{-1}(\mathbb{R})$ can be parametrized by polar coordinates (9). Further, a numerical computation indicates that no critical point of $b$ lies on the Jordan curve in the upper half-plane. The real critical points of $b$ closest to the origin are $z_{1} \approx 1.077904$ and $z_{2} \approx-0.844126$ and the corresponding critical values are $b\left(z_{1}\right) \approx 14.9641$ and $b\left(z_{2}\right) \approx-22.0915$. So the limiting measure $\mu$ should be supported approximately on the interval $[-22.09,14.96]$ which is in agreement with the numerical results obtained by an implementation of the algorithm for the computation of $\Lambda(b)$ suggested in [3]. The density of $\mu$ is plotted in Fig. 5.

\subsubsection{Example 5}

For an illustration of Theorem 13 which allows having nonreal critical points of $b$ on the arc of Jordan curve in $b^{-1}(\mathbb{R})$, we consider the symbol

$$
b(z)=\frac{1}{z^{3}}+\frac{1}{z^{2}}+\frac{1}{z}+z+z^{2}+z^{3} .
$$

The corresponding Toeplitz matrix $T(b)$ is self-adjoint, and hence the Jordan curve in $b^{-1}(\mathbb{R})$ is the unit circle. The function $b$ has two critical points with positive imaginary part on the unit circle, namely

$$
\begin{aligned}
& z_{1}:=\frac{1}{6}(-1+\sqrt{7}+\mathrm{i} \sqrt{2(14+\sqrt{7})}) \approx 0.274292+0.961646 \mathrm{i}, \\
& z_{2}:=\frac{1}{6}(-1-\sqrt{7}+\mathrm{i} \sqrt{2(14-\sqrt{7})}) \approx-0.607625+0.794224 \mathrm{i},
\end{aligned}
$$




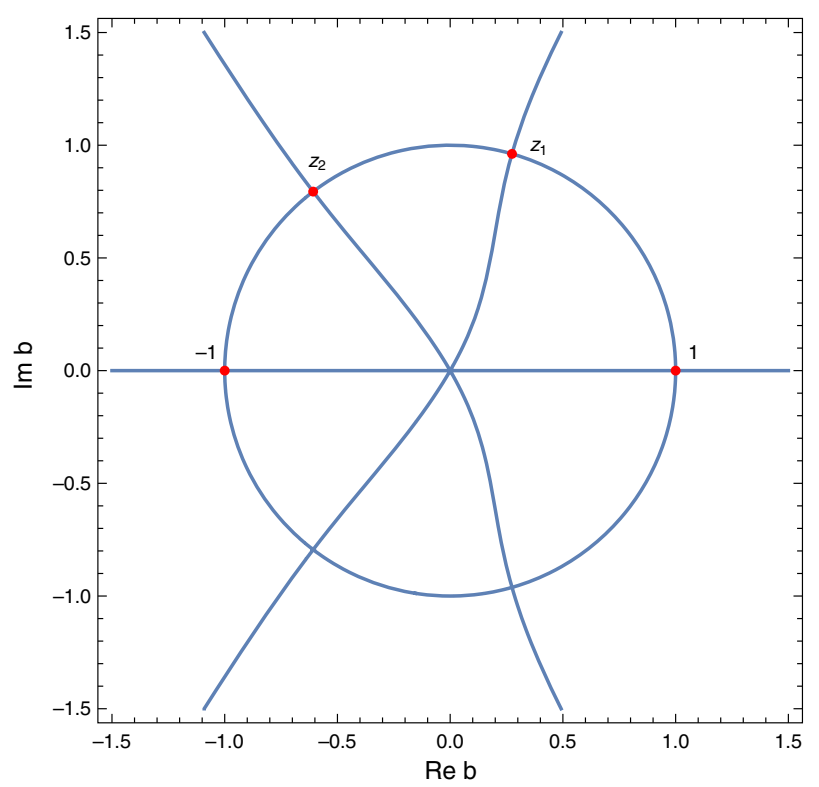

Fig. 6 Illustration for Example 5: The plot of the net of $b(z)=1 / z^{3}+1 / z^{2}+1 / z+z+z^{2}+z^{3}$

and the corresponding values are

$$
b\left(z_{1}\right) \approx-2.63113 \text { and } b\left(z_{2}\right) \approx 0.112612
$$

see Fig. 6. Theorem 13 tells us that each arc of the unit circle between two critical points gives rise to a measure. Labeling the measures in agreement with Theorem 13 (starting at 1 and traversing the arcs of the unit circle in the counterclockwise direction), we get measures $\mu_{1}, \mu_{2}$, and $\mu_{3}$, which, since $b(1)=6$ and $b(-1)=-2$, are supported approximately on the intervals $[-2.63,6],[-2.63,0.11]$, and $[-2,0.11]$, respectively. The limiting measure then equals $\mu=\mu_{1}+\mu_{2}+\mu_{3}$. The illustration of the corresponding densities are given in Figs. 7 and 8 .

The graph of the density of $\mu$ suggests that the eigenvalues of $T_{n}(b)$ cluster with higher density to the left of the point 0.11 which has also been observed numerically, see Fig. 9.

\subsubsection{Breaking the Reality of $\Lambda(b)$}

Our final plots are devoted to an illustration of the connection between the presence of a Jordan curve in $b^{-1}(\mathbb{R})$ and the reality of $\Lambda(b)$. We go back to the Example 4 once again and introduce an additional parameter $\alpha \in \mathbb{R}$ at $z^{2}$, getting the symbol

$$
b(z)=\frac{1}{z^{3}}-\frac{1}{z^{2}}+\frac{7}{z}+9 z+\alpha z^{2}+2 z^{3}-z^{4} .
$$




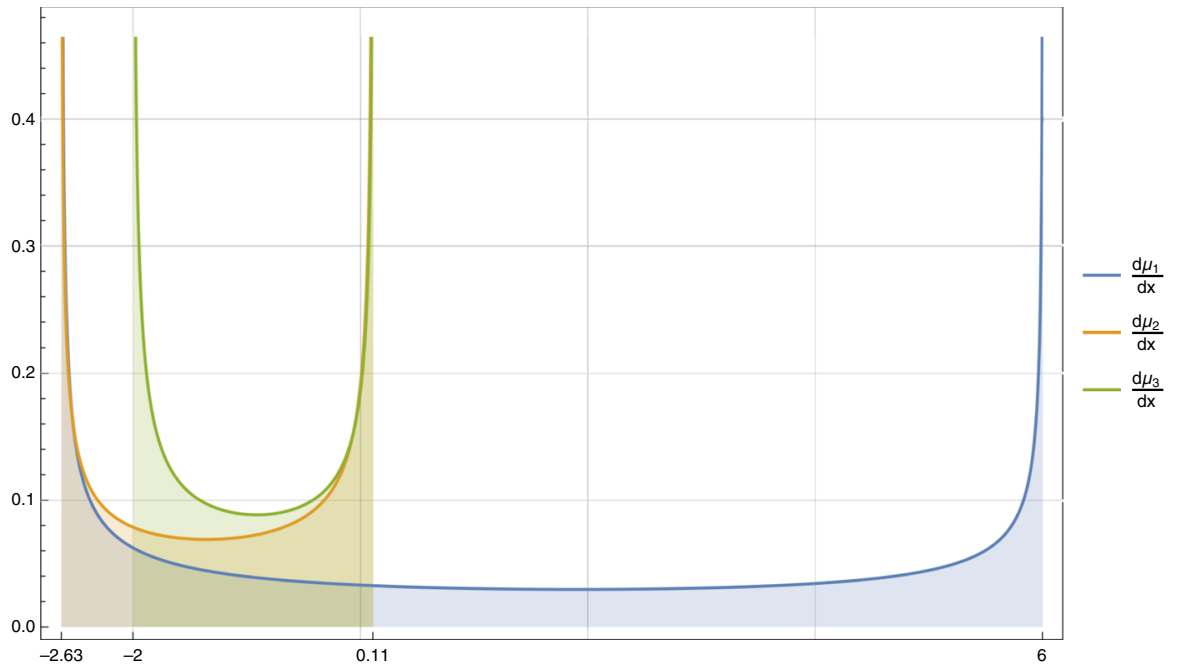

Fig. 7 Illustration for Example 5: The plots of densities of $\mu_{1}, \mu_{2}$, and $\mu_{3}$ (Color figure online)

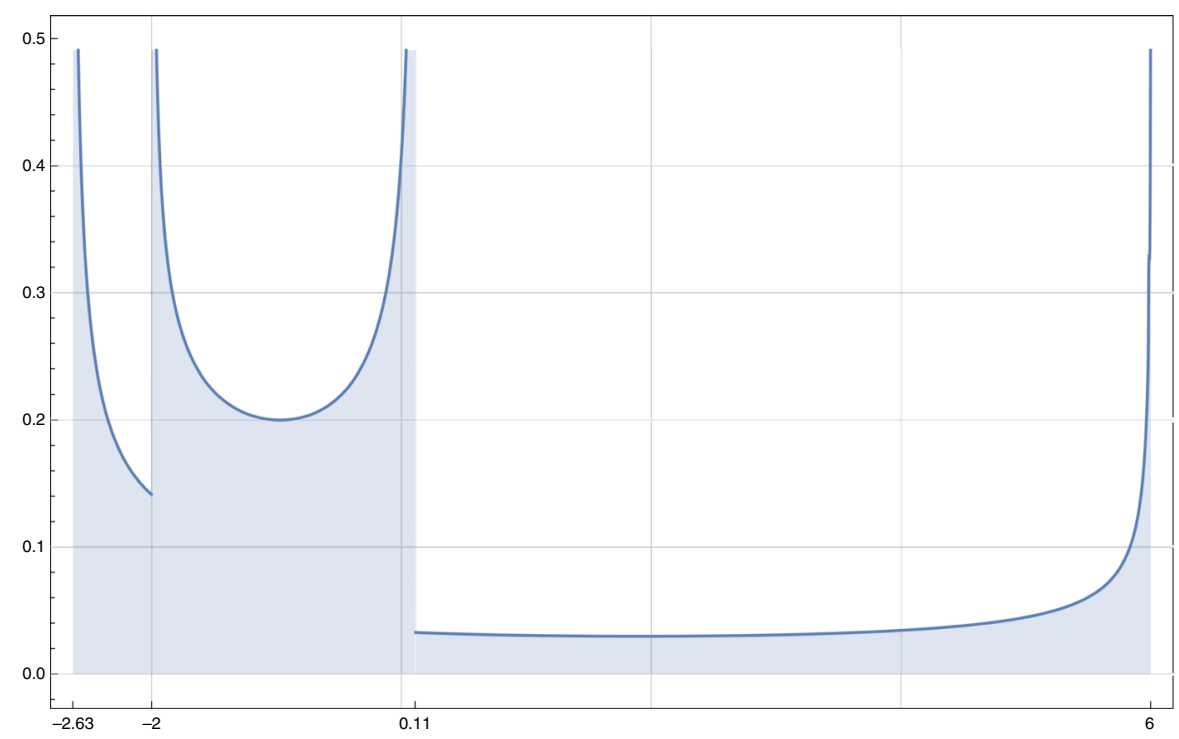

Fig. 8 Illustration for Example 5: The plot of the density of $\mu$

In Fig. 10, it is shown that, as the parameter $\alpha$ increases from -2 to 2 , the Jordan curve in $b^{-1}(\mathbb{R})$ gets "destroyed" by an incoming curve from the net of $b$. At the same time, $\Lambda(b)$ changes from a real interval to a set with nonreal values. The set $\Lambda(b)$ is plotted by using the algorithm from [3]. 


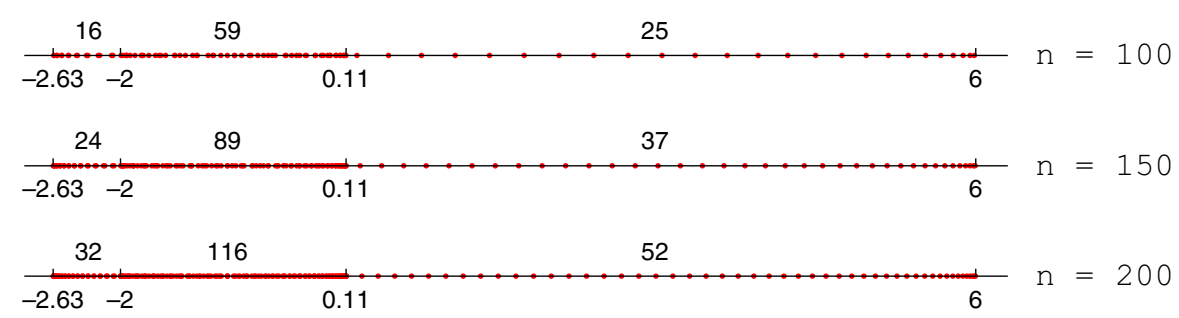

Fig. 9 Illustration for Example 5: The distribution of eigenvalues of $T_{n}(b)$ for $n \in\{100,150,200\}$ in the interval $[-2.63,6]$. The numbers above the segments indicate the number of eigenvalues within the respective segment
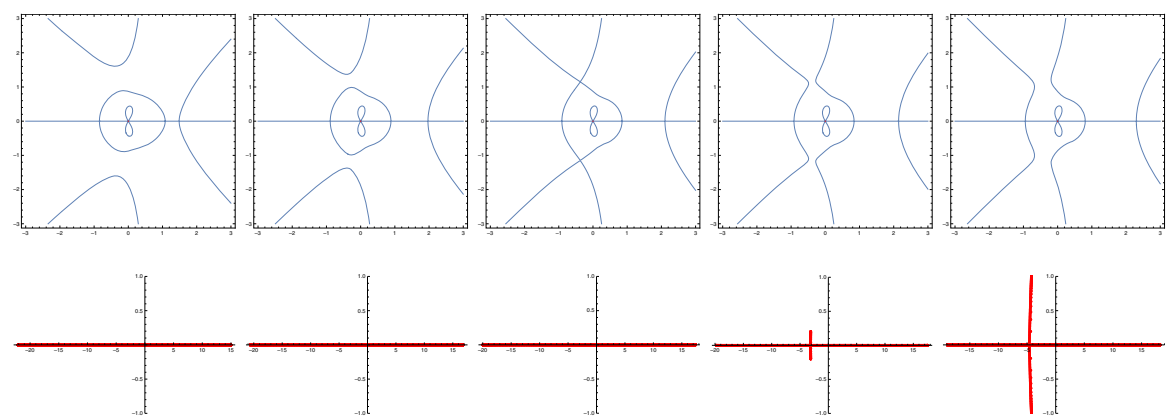

Fig. 10 The plots of the net of $b(z)=1 / z^{3}-1 / z^{2}+7 / z+9 z+\alpha z^{2}+2 z^{3}-z^{4}$ (above) and corresponding plots of $\Lambda(b)$ (below) for $\alpha \in\{-2,0,0.77,1,2\}$

Acknowledgements The authors wish to express their gratitude to Professor A. B. J. Kuijlaars for pointing out an error in the first version of the manuscript and to Professor M. Duits for his interest in the topic. Finally, the authors want to thank both anonymous referees for their suggestions resulting in several improvements of the exposition and for the reference to the papers of Day [10,11].

Open Access This article is distributed under the terms of the Creative Commons Attribution 4.0 International License (http://creativecommons.org/licenses/by/4.0/), which permits unrestricted use, distribution, and reproduction in any medium, provided you give appropriate credit to the original author(s) and the source, provide a link to the Creative Commons license, and indicate if changes were made.

\section{Appendix}

The following two simple observations are used for an explanation of the structure of the set $b^{-1}(\mathbb{R})$ in the beginning of Sect.2. They follow readily from the maximum modulus principle and open mapping theorem, but we provide them with proofs for the reader's convenience.

Lemma 24 Let $f$ be an analytic function in a neighborhood of a disk $D \subset \mathbb{C}$. Put $\Gamma:=\{z \in D \mid \operatorname{Im} f(z)=0\}$ and suppose that $\Gamma \neq \emptyset$ and $D \backslash \Gamma$ is connected. Then $f$ is constant on $D$.

Remark 25 As an example referring to the concrete situation studied in Sect.2, the set $\Gamma$ can be an arc of a curve that does not start and terminate on the boundary of $D$. 
Proof Since $f$ is analytic, $h:=\operatorname{Im} f$ is harmonic. Further, function $h$ does not change sign on the connected set $D \backslash \Gamma$ by the continuity of $h$. Let us suppose, without loss of generality, that $h<0$ on $D \backslash \Gamma$. Since $h$ attains its maximum in $D$ at a point from $\Gamma$, the maximum modulus principle for harmonic functions implies that $h$ is constant on $D$. In this case, $h=0$ on $D$, and hence $f$ maps the disk $D$ into $\mathbb{R}$. By the open mapping theorem for analytic functions, such $f$ has to be constant.

Lemma 26 Let $f$ be an analytic function on a neighborhood in a bounded nonempty region $\Omega \subset \mathbb{C}$ such that $\operatorname{Im} f(z)=0$ for all $z \in \partial \Omega$. Then $f$ is constant on $\Omega$.

Proof Define $g_{ \pm}:=e^{ \pm \mathrm{i} f}$. By the assumptions, $\left|g_{ \pm}(z)\right|=1$ for all $z \in \partial \Omega$. By the maximum modulus principle for analytic functions, $\left|g_{ \pm}(z)\right| \leq 1$ for all $z \in \Omega$. Since $\left|g_{ \pm}(z)\right|=e^{\mp \operatorname{Im} f(z)}$, the latter inequality implies that $\mp \operatorname{Im} f(z) \leq 0$ for all $z \in \Omega$. Consequently, $\operatorname{Im} f=0$ on $\Omega$, and hence $f$ maps $\Omega$ into $\mathbb{R}$. By the open mapping theorem for analytic functions, such $f$ is a constant function.

The following determinant formula presented in Lemma 27 was used in Sect. 4.1. It can be deduced from the well-known identity for the Hankel transform of the sequence of central binomial coefficients. Although the derivation is elementary, it is not completely straightforward, and therefore we provide it along with its proof.

Lemma 27 Let $n \in \mathbb{N}$ and $H_{n}$ be the Hankel matrix of the form (11) with elements given by (32); then one has

$$
\operatorname{det} H_{n}=2^{n-1} a^{n(n-1) / 2} \text {. }
$$

Proof First note that, if $H_{n}$ and $G_{n}$ are two Hankel matrices with $\left(H_{n}\right)_{i, j}=h_{i+j-2}$ and $\left(G_{n}\right)_{i, j}=g_{i+j-2}$ such that $h_{m}=\alpha^{m} g_{m}$, for some $\alpha \in \mathbb{C}$, then $H_{n}=$ $D_{n}(\alpha) G_{n} D_{n}(\alpha)$, where $D_{n}(\alpha)=\operatorname{diag}\left(1, \alpha, \ldots, \alpha^{n-1}\right)$. Therefore it suffices to verify the statement for $a=1$.

Let $k, n \in \mathbb{N}$ and $G_{n}^{(k)}$ be the Hankel matrix whose entries are given by

$$
\left(G_{n}^{(k)}\right)_{i, j}=\left(\begin{array}{c}
2(i+j+k-2) \\
i+j+k-2
\end{array}\right) \text { for } i, j \in\{1,2, \ldots, n\} .
$$

The formula for det $G_{n}^{(k)}$ is well known and, in particular, one has

$$
\operatorname{det} G_{n}^{(1)}=2 \operatorname{det} G_{n}^{(0)}=2^{n}, \quad \forall n \in \mathbb{N},
$$

see, for example, $[1,16]$.

Next we make use of the direct sum decomposition $\mathbb{C}^{n}=\mathbb{C}_{\text {odd }}^{n} \oplus \mathbb{C}_{\text {even }}^{n}$, where

$$
\mathbb{C}_{\text {odd }}^{n}:=\operatorname{span}\left\{e_{2 j-1} \mid 1 \leq 2 j-1 \leq n\right\} \quad \text { and } \quad \mathbb{C}_{\text {even }}^{n}:=\operatorname{span}\left\{e_{2 j} \mid 1 \leq 2 j \leq n\right\} .
$$

Since $h_{2 m-1}=0$ for all $m \in \mathbb{N}$, both subspaces $\mathbb{C}_{\text {odd }}^{n}$ and $\mathbb{C}_{\text {even }}^{n}$ are $H_{n}$-invariant, i.e., $H_{n} \mathbb{C}_{\text {odd }}^{n} \subset \mathbb{C}_{\text {odd }}^{n}$ and $H_{n} \mathbb{C}_{\text {even }}^{n} \subset \mathbb{C}_{\text {even }}^{n}$. The matrix $H_{n}$ decomposes accordingly as

$$
H_{n}=H_{n}^{\text {odd }} \oplus H_{n}^{\text {even }}
$$


where

$$
H_{n}^{\text {odd }}=G_{\lfloor(n+1) / 2\rfloor}^{(0)} \quad \text { and } \quad H_{n}^{\text {even }}=G_{\lfloor n / 2\rfloor}^{(1)} \text {. }
$$

Thus, by using formulas in (45), one obtains

$$
\operatorname{det} H_{n}=\operatorname{det}\left(G_{\lfloor(n+1) / 2\rfloor}^{(0)}\right) \operatorname{det}\left(G_{\lfloor n / 2\rfloor}^{(1)}\right)=2^{n-1} .
$$

\section{References}

1. Aigner, M.: Catalan-like numbers and determinants. J. Combin. Theory Ser. A 87(1), 33-51 (1999)

2. Akhiezer, N.I.: Elements of the theory of elliptic functions, vol. 79 of Translations of Mathematical Monographs. American Mathematical Society, Providence, RI, 1990. Translated from the second Russian edition by H. H. McFaden

3. Beam, R.M., Warming, R.F.: The asymptotic spectra of banded Toeplitz and quasi-Toeplitz matrices. SIAM J. Sci. Comput. 14(4), 971-1006 (1993)

4. Böttcher, A., Grudsky, S.M.: Can spectral value sets of Toeplitz band matrices jump? Linear Algebra Appl. 351/352, 99-116 (2002). (Fourth special issue on linear systems and control)

5. Böttcher, A., Grudsky, S.M.: Spectral Properties of Banded Toeplitz Matrices. Society for Industrial and Applied Mathematics (SIAM), Philadelphia (2005)

6. Caliceti, E., Cannata, F., Graffi, S.: Perturbation theory of $\mathcal{P} \mathcal{T}$-symmetric Hamiltonians. J. Phys. A Math. Gen. 39, 10019-10027 (2006)

7. Chihara, T.S.: An Introduction to Orthogonal Polynomials. Mathematics and Its Applications, vol. 13. Gordon and Breach Science Publishers, New York (1978)

8. Coussement, E., Coussement, J., Van Assche, W.: Asymptotic zero distribution for a class of multiple orthogonal polynomials. Trans. Am. Math. Soc. 360(10), 5571-5588 (2008)

9. Davies, E.B.: Linear Operators and Their Spectra. Cambridge Studies in Advanced Mathematics, vol. 106. Cambridge University Press, Cambridge (2007)

10. Day, K.: Michael Toeplitz matrices generated by the Laurent series expansion of an arbitrary rational function. Trans. Am. Math. Soc. 206, 224-245 (1975)

11. Day, K.: Michael Measures associated with Toeplitz matrices generated by the Laurent expansion of rational functions. Trans. Am. Math. Soc. 209, 175-183 (1975)

12. Denisov, S.A.: On Rakhmanov's theorem for Jacobi matrices. Proc. Am. Math. Soc. 132(3), 847-852 (2004)

13. Duits, M., Kuijlaars, A.B.J.: An equilibrium problem for the limiting eigenvalue distribution of banded Toeplitz matrices. SIAM J. Matrix Anal. Appl. 30(1), 173-196 (2008)

14. Eğecioğlu, Ö., Redmond, T., Ryavec, C.: Evaluation of a special Hankel determinant of binomial coefficients. In: Fifth Colloquium on Mathematics and Computer Science. Discrete Mathematics and Theoretical Computer Science Proceedings, AI, Nancy, pp. 251-267 (2008)

15. Eremenko, A., Gabrielov, A.: Rational functions with real critical points and the B. and M. Shapiro conjecture in real enumerative geometry. Ann. Math. (2) 155(1), 105-129 (2002)

16. Garcia Armas, M., Sethuraman, B.A.: A note on the Hankel transform of the central binomial coefficients. J. Integer Seq. 11, 3 (2008)

17. Giordanelli, I., Graf, G.M.: The real spectrum of the imaginary cubic oscillator: an expository proof. Ann. Henri Poincaré 16, 99-112 (2015)

18. Helffer, B.: Spectral Theory and Its Applications. Cambridge Studies in Advanced Mathematics, vol. 139. Cambridge University Press, Cambridge (2013)

19. Hirschman Jr., I.I.: The spectra of certain Toeplitz matrices. Ill. J. Math. 11, 145-159 (1967)

20. Kac, M.: Toeplitz matrices, translation kernels and a related problem in probability theory. Duke Math. J. 21, 501-509 (1954) 
21. Koekoek, R., Lesky, P.A., Swarttouw, R.F.: Hypergeometric Orthogonal Polynomials and Their $q$ Analogues. Springer Monographs in Mathematics. Springer, Berlin, With a foreword by Tom H. Koornwinder (2010)

22. Kuijlaars, A.B.J.: Chebyshev quadrature for measures with a strong singularity. In: Proceedings of the International Conference on Orthogonality, Moment Problems and Continued Fractions (Delft, 1994) , vol. 65, pp. 207-214 (1995)

23. Langer, H., Tretter, C.: A Krein space approach to PT-symmetry. Czech. J. Phys. 54, 1113-1120 (2004)

24. Mityagin, B., Siegl, P.: Root system of singular perturbations of the harmonic oscillator type operators. Lett. Math. Phys. 106(2), 147-167 (2016)

25. Nevai, P.G.: Orthogonal polynomials. Mem. Am. Math. Soc. 18, 213 (1979)

26. Prudnikov, A.P., Brychkov, Y.A., Marichev, O.I.: Integrals and Series, vol. 3. Gordon and Breach Science Publishers, New York. More special functions. Translated from the Russian by G. G. Gould (1990)

27. Schmidt, P., Spitzer, F.: The Toeplitz matrices of an arbitrary Laurent polynomial. Math. Scand. 8, 15-38 (1960)

28. Shin, K.C.: On the reality of the eigenvalues for a class of $\mathscr{P} \mathscr{T}$-symmetric oscillators. Commun. Math. Phys. 229(3), 543-564 (2002)

29. Shohat, J.A., Tamarkin, J.D.: The Problem of Moments. American Mathematical Society Mathematical Surveys, vol. I. American Mathematical Society, New York (1943)

30. Siegl, P., Štampach, F.: Spectral analysis of Jacobi matrices associated with Jacobian elliptic functions. Oper. Matrices 11(4), 901-928 (2017)

31. Simon, B.: The classical moment problem as a self-adjoint finite difference operator. Adv. Math. 137(1), 82-203 (1998)

32. Teschl, G.: Jacobi Operators and Completely Integrable Nonlinear Lattices. Mathematical Surveys and Monographs, vol. 72. American Mathematical Society, Providence (2000)

33. Trefethen, L.N., Embree, M.: Spectra and Pseudospectra: The Behavior of Nonnormal Matrices and Operators. Princeton University Press, Princeton (2005)

34. Ullman, J.L.: A problem of Schmidt and Spitzer. Bull. Am. Math. Soc. 73, 883-885 (1967)

35. Weir, J.: An indefinite convection-diffusion operator with real spectrum. Appl. Math. Lett. 22(2), 280-283 (2009)

36. Wimp, J.: Explicit formulas for the associated Jacobi polynomials and some applications. Can. J. Math. 39(4), 983-1000 (1987) 